\title{
Die Repräsentation der Bürgerinnen und Bürger durch organisierte Interessen in Deutschland
}

\author{
Dominic Pakull (iD · Felix Goldberg • Patrick Bernhagen
}

Online publiziert: 15. Juni 2020

(C) Der/die Autor(en) 2020, korrigierte Publikation 2020

Zusammenfassung In dieser Studie analysieren wir das Potenzial organisierter Interessen als Transmissionsriemen zwischen der Bevölkerung und politischen Institutionen im parlamentarischen System der Bundesrepublik zu fungieren. Wir verwenden Daten aus Experteninterviews mit Lobbyistinnen und Lobbyisten und einer repräsentativen Bevölkerungsumfrage, um die Kongruenz zwischen den Themenfeldern zu ermitteln, welche die Bürgerinnen und Bürger und organisierten Interessen in Deutschland jeweils als wichtig ansehen. Ferner analysieren wir, ob sich die Kongruenz hinsichtlich der öffentlichen Salienz des Themas sowie des soziökonomischen Status oder der politischen Einstellungen der Bürgerinnen und Bürger unterscheidet. Dabei differenzieren wir zwischen Unternehmer- und Nichtunternehmerinteressen. Die Ergebnisse deuten auf eine moderate Themenkongruenz zwischen den Agenden der Bürgerinnen und Bürger und der organisierten Interessen hin. Diese Repräsentationsleistung wird jedoch überwiegend von Nichtunternehmerorganisationen erfüllt, die verstärkt an Themen arbeiten, die für die Bevölkerung salient sind. Ferner sind Bürgerinnen und Bürger mit extremeren Einstellungen besser repräsentiert als jene, die sich in der ideologischen Mitte befinden. Hingegen zeigen sich keine Unterschiede hinsichtlich des Einkommens und Geschlechts oder zwischen Ost- und Westdeutschland.

Schlüsselwörter Lobbyismus · Interessengruppen · Kongruenz · Öffentliche Meinung · Unternehmer und Politik

D. Pakull $(\bowtie) \cdot$ F. Goldberg $\cdot$ P. Bernhagen Institut für Sozialwissenschaften, Universität Stuttgart, Breitscheidstraße 2, 70174 Stuttgart, Deutschland E-Mail: dominic.pakull@sowi.uni-stuttgart.de 


\section{The Representation of Citizens by Organized Interests in Germany}

Abstract In this study, we analyse the potential of organized interests to act as a conveyer belt between the population and political institutions in the German parliamentary system. Using data from expert interviews with lobbyists and a representative citizen survey we assess the congruence between the issue areas that matter most to the public and to organized interests in Germany. We further analyse whether the congruence differs with regard to the public salience of an issue and to citizens' socioeconomic status or their political attitudes. Therefore, we differentiate between business and non-business interests. The results indicate a modest congruence of issues between the agendas of the public and of organized interests. However, this representative function is performed predominantly by non-business interest organizations that work on the more publicly salient topics. Furthermore, citizens with more extreme attitudes are better represented than those in the ideological centre. In contrast, there are no differences with regard to income and gender, or between East and West Germany.

Keywords Lobbying · Interest groups · Congruence $\cdot$ Public opinion · Business and politics

\section{Einleitung}

Im Laufe der letzten beiden Jahrzehnte mussten westliche Demokratien einen beachtlichen Niedergang des Vertrauens der Bürgerinnen und Bürger in politische Institutionen und Parteien verzeichnen (Citrin und Stoker 2018; Newton 2012; Norris 2011). Dieser drückt sich nicht zuletzt in weitreichenden Veränderungen der westeuropäischen Parteiensysteme aus, insbesondere dem Aufstieg neuer, zumeist rechtspopulistischer „Anti-Parteien-Parteien“ (Mudde 1996), aber auch in sozialen Bewegungen wie den ,gilets jaunes“ (Gelbwesten) in Frankreich. Der Vertrauensverlust könnte berechtigt sein. So haben Studien v. a. im Kontext der USA (Bartels 2016; Gilens 2012; Gilens und Page 2014), aber auch in anderen westlichen Demokratien (Elsässer et al. 2017; Giger et al. 2012; Peters und Ensink 2015) gezeigt, dass staatliches Handeln den Präferenzen der Einkommensstarken in größerem Umfang entspricht als denen der Einkommensschwachen. Somit könnte die Repräsentativität des politischen Systems verzerrt sein. Zugleich wird zivilgesellschaftlichen Organisationen bereits seit langem die Fähigkeit zugesprochen, Mängel der Repräsentation durch Parteien zu kompensieren (Richardson 1995). So wird argumentiert, dass organisierte Interessen neben Parteien als Transmissionsriemen fungieren und gesellschaftliche Präferenzen in das politische System übertragen können (Berry und Wilcox 2018; Williams 2000; Easton 1971; Truman 1951).

Während jedoch eine Reihe von Studien zur repräsentativen Funktion von Parteien vorhanden sind (Romeijn 2018; Spoon und Klüver 2014; Carmines und Stimson 1986), gibt es nach derzeitigem Stand nur wenige empirische Untersuchungen, die sich mit dem repräsentativen Potenzial von organisierten Interessen auseinandersetzen (Bevan und Rasmussen 2017; Kimball et al. 2012; Rasmussen et al. 2014). Diese 
wenigen Analysen zeichnen zudem ein gemischtes Bild hinsichtlich der Repräsentativität von organisierten Interessen. So konnten einerseits Bevan und Rasmussen (2017) zeigen, dass sich mit der Zahl der in einem Politikfeld aktiven Gruppen auch die Wahrscheinlichkeit erhöht, dass Themen, die für die Öffentlichkeit wichtig sind, auf die Tagesordnung der Regierung gelangen. Andere Studien haben hingegen herausgefunden, dass es nur eine geringe Schnittmenge zwischen den Agenden von organisierten Interessen und der Öffentlichkeit gibt (z. B. Kimball et al. 2012). Arbeiten, welche die Verknüpfung zwischen den politisch aktivsten Interessengruppen auf der einen sowie Bürgerinnen und Bürgern und Parteien auf der anderen Seite untersuchen, deuten darauf hin, dass organisierte Interessen als Transmissionsriemen zwischen Bürgerinnen und Bürgern und politischen Institutionen fungieren können (Klüver 2018, 2015). In dieser Studie gehen wir über den bisherigen Forschungsstand hinaus und untersuchen zum ersten Mal systematisch die Themenkongruenz zwischen den Agenden von Bürgerinnen und Bürgern und organisierten Interessen. Wir analysieren damit das repräsentative Potenzial der organisierten Interessen in der Bundesrepublik und berücksichtigen dabei Organisationen mit unternehmerischem und nichtunternehmerischem Interesse. Unter organisierten Interessen verstehen wir alle kollektiven Akteure, die ihre Präferenzen im politischen System zu vertreten suchen, ohne dabei Regierungsämter oder parlamentarische Vertretung durch Wahlen anzustreben. Somit betrachten wir neben Verbänden, die Individuen, Unternehmen oder andere Verbände als Mitglieder haben können, auch Unternehmen sowie berufsständische Vereinigungen als organisierte Interessen (Baumgartner und Leech 1998; Schlozman und Tierney 1986).

Wir legen erstens den Fokus darauf, in welchem Ausmaß die Themen, die der Bevölkerung wichtig sind, von organisierten Interessen bearbeitet werden. Zweitens analysieren wir, ob alle Bürgerinnen und Bürger in etwa gleich gut oder schlecht von organisierten Interessen repräsentiert werden, oder ob Unterschiede in Bezug auf soziökonomische Faktoren oder ideologische Einstellungen der Bürgerinnen und Bürger bestehen. Dabei betrachten wir auch, ob die Repräsentationsleistung zwischen unternehmerischen und nichtunternehmerischen Interessen schwankt. Unsere Analyse stellt damit einen notwendigen ersten Schritt dar, um sich der Frage anzunähern, inwiefern organisierte Interessen in einer Konsensdemokratie mit parlamentarischem Regierungssystem (Lijphart 2012) als Transmissionsriemen zwischen Bürgerinnen und Bürgern und Staat fungieren können (Easton 1971; Truman 1951). Eine bislang einzigartige Verknüpfung von repräsentativen Umfragedaten mit einer großen Zahl teilstandardisierter Experteninterviews mit Lobbyistinnen und Lobbyisten ermöglicht es uns, die politischen Agenden von organisierten Interessen und Bürgerinnen und Bürgern systematisch zu vergleichen. Aus insgesamt 119 Interviews konnten wir 797 Themen ermitteln, die zudem einen guten Einblick in die Prioritäten der Lobbylandschaft in Deutschland zum Zeitpunkt der Interviews geben.

Im weiteren Verlauf des Artikels gehen wir wie folgt vor: Zunächst entwickeln wir im Lichte der Literatur theoretisch begründete Erwartungen bezüglich der Repräsentationsleistung organisierter Interessen (Abschn. „Die repräsentative Funktion organisierter Interessen“). Anschließend beschreiben wir die Quellen und Erhebung unserer Daten (Abschn. „Daten“). In einer bivariaten empirischen Analyse betrachten wir sodann die Kongruenz von Gruppen und Bevölkerung auf aggregierter Ebene 
(Abschn. ,Themenkongruenz zwischen Bürgerinnen, Bürgern und organisierten Interessen"). Dieser eher explorative Analyseschritt vermittelt einen ersten Eindruck vom Grad der Themenkongruenz von Bürgerinnen und Bürgern und organisierten Interessen und veranschaulicht, welche Themen für welche Akteure besonders dringlich sind. Im Rahmen der anschließenden multivariaten Analyse testen wir unsere Hypothesen (Abschn. „Multivariate Analyse: Individuelle Themenkongruenz und Repräsentation durch organisierte Interessen"). Hier untersuchen wir auf der individuellen Ebene, welche soziökonomischen und ideologischen Faktoren die Repräsentation der Bürgerinnen und Bürger durch organisierte Interessen beeinflussen, wobei wir zwischen Unternehmer- und Nichtunternehmerinteressen differenzieren. Abschießend ziehen wir Schlussfolgerungen aus unseren Befunden und diskutieren diese hinsichtlich der Implikationen für die bestehende und weitere Forschung zur Repräsentation und Interessenvermittlung (Abschn. „Diskussion und Schlussfolgerungen“").

\section{Die repräsentative Funktion organisierter Interessen}

In modernen Demokratien wird Repräsentation über den Selektionsmechanismus der Wahlen sowie die Responsivität von Politikerinnen und Politkern gegenüber den Bürgerinnen und Bürgern zwischen den Wahlen ermöglicht (Dahl 1971). Neben der Responsivität wird vor allem die ideologische Kongruenz zwischen Bürgerinnen und Bürgern und politischen Eliten als Manifestation funktionierender Repräsentation verstanden (Golder und Ferland 2018). Kongruenz und Responsivität sind konzeptuell eng miteinander verwandt, unterscheiden sich jedoch durch ihre zeitliche Dimension. Kongruenz misst, inwieweit die Handlungen der politischen Eliten mit den Interessen der Bürgerinnen und Bürger zu einem gegebenen Zeitpunkt in Einklang sind, wohingegen sich die Responsivität darauf bezieht, wie die Eliten ihr Handeln an den Bedürfnissen der Bürgerinnen und Bürger orientieren. Kongruenz kann sich wiederum auf die Themenprioritäten (Jones und Baumgartner 2004; Monroe 1979; Verba und Nie 1972), die Positionen in Bezug auf diese Themen (Wlezien 2017; Lax und Phillips 2012; Erikson et al. 1993) oder auf beides beziehen (Stecker und Tausendpfund 2016). In der vorliegenden Studie konzentrieren wir uns auf Themenkongruenz von Bürgerinnen und Bürgern und organisierten Interessen. Unter Themenkongruenz verstehen wir das Ausmaß, in dem die von den Bürgerinnen und Bürgern genannten Themen auf den Agenden der organisierten Interessen vertreten sind. Dies ermöglicht es uns, die Repräsentationsleistung von organisierten Interessen zu eruieren.

Um als intermediäre Organisationen zu fungieren und gesellschaftliche Präferenzen ins politische System einzuspeisen (Easton 1971; Truman 1951), müssen organisierte Interessen ein gewisses Maß an Themenkongruenz mit den Bürgerinnen und Bürgern aufweisen. Dies kann einerseits als Kongruenz einzelner Interessengruppen mit ihren jeweiligen Mitgliedern oder Unterstützern der Fall sein oder als Kongruenz des Interessengruppensystems in seiner Gesamtheit mit der Bevölkerung. Bisherige Studien zu positioneller Kongruenz haben untersucht, ob es eine ideologische Korrelation zwischen den Präferenzen von Bürgerinnen und Bürgern und den Inhalten von 
konkreten Politiken gibt (Wlezien 2017; Erikson et al. 1993; Kuklinski 1978), oder ob ein positiver Zusammenhang zwischen den Ausgabenwünschen der Bürgerinnen und Bürger in ausgewählten Politikfeldern und den entsprechenden Haushaltstiteln besteht (Wlezien und Soroka 2012; Canes-Wrone und Shotts 2004). Früh haben Forscher auch die Themenkongruenz in den Blick genommen. So untersuchen etwa Verba und Nie (1972, S. 310) die Kongruenz der Prioritäten von Bürgerinnen und Bürgern und Politikerinnen und Politikern auf kommunaler Ebene und gleichen dabei die Übereinstimmung der jeweils als wichtigstes Problem angesehenen Themen ab. Dabei ist die Kongruenz mit den kommunalen Entscheidungsträgern umso gröBer, je stärker Bürgerinnen und Bürger auf kommunaler Ebene partizipieren (Verba und Nie 1972, S. 305). Jones und Baumgartner (2004) untersuchen, inwiefern die Themen, welche die Bürgerinnen und Bürger als am wichtigsten erachten, sich in der Arbeit der US-Regierung widerspiegeln. Bis heute jedoch haben sich nur wenige Studien mit der repräsentativen Funktion von organisieren Interessen beschäftigt (Bevan und Rasmussen 2017; Klüver 2015; Rasmussen et al. 2014).

In einer Studie zur Umweltpolitik in den USA hat Agnone (2007) herausgefunden, dass sich öffentliche Meinung und Lobbying gegenseitig verstärken können. So erhöht das Lobbying von Umweltgruppen die Salienz des Themas für Politikerinnen und Politiker in einem Feld, das - im konkreten Fallbeispiel - auch für die Öffentlichkeit wichtig ist. Im europäischen Kontext haben Rasmussen et al. (2014) eine Verbindung zwischen öffentlicher Meinung und Mobilisierung von Interessengruppen in dem Sinne festgestellt, dass die Interessengruppenaktivität im Konsultationsverfahren der Europäischen Kommission ansteigt, wenn ein Thema unter den Bürgerinnen und Bürgern salient ist. Für westeuropäische Länder konnten Flöthe und Rasmussen (2019) bei den von ihnen untersuchten Themen ein gewisses Maß an positioneller Kongruenz zwischen Bürgerinnen und Bürgern und Interessengruppen nachweisen. Boräng et al. (2018) befinden in ihrer vergleichenden Studie zu fünf EU-Ländern, dass Interessengruppen in ihrer Gesamtheit die Verteilung ideologischer Präferenzen in der Bevölkerung besser widerspiegeln als politische Parteien. Dies gilt insbesondere in westeuropäischen Ländern wie Belgien und Schweden, in denen die Parteien Bürgerinnen und Bürgern, die ökonomisch links, aber bei sozialkulturellen Themen eher autoritär eingestellt sind, häufig kein Angebot machen (Jungar und Jupskas 2014; de Lange 2007).

Im deutschen Kontext haben ebenfalls nur wenige Studien untersucht, in welchem Ausmaß Interessengruppen als Transmissionsriemen zwischen Bürgerinnen und Bürgern und dem Staat fungieren können. So hat Klüver (2015) einen Bandwagon-Effekt nachgewiesen, dem gemäß umso mehr Interessengruppen in einem Politikfeld aktiv werden, je salienter ein Thema für die Öffentlichkeit wird (Klüver 2015). In einer weiteren Untersuchung hat Klüver (2018) gezeigt, dass die Parteien ihre Agenden dem Grad der Mobilisierung von Interessengruppen und den Forderungen der Wählerinnen und Wähler anpassen. Dabei sind Interessengruppen erfolgreicher als die Wählerinnen und Wähler darin, die politischen Prioritäten von Parteien zu beeinflussen (Klüver 2018).

Da die Repräsentantinnen und Repräsentanten organisierter Interessen nicht primär für Wahlen zu öffentlichen Ämtern kandidieren, müssen sie sich nicht in demselben Maße wie politische Parteien an den Präferenzen der Bevölkerung orientieren. 
Stattdessen sind sie bei der Auswahl der Themen, auf die sie einen Schwerpunkt legen wollen, vorrangig ihren Mitgliedern gegenüber verantwortlich (Halpin et al. 2018). Dies könnte dazu führen, dass organisierte Interessen zum einen an Themen arbeiten, die weniger salient für die breitere Bevölkerung sind. Zum anderen unterliegen diese weniger Schwankungen, da die Interessen der Gruppenmitglieder relativ zu tagespolitischen Entwicklungen oder der öffentlichen Meinung recht stabil sind (Kimball et al. 2012). So bezeichnet etwa der Begriff des „niche lobbying“ die politische Arbeit an Themen, die für einzelne organisierte Interessen von großer und oftmals auch dauerhafter Bedeutung sind, für welche die breitere Öffentlichkeit jedoch kaum Interesse aufbringt (Baumgartner und Leech 2001). In dem Maß, in dem organisierte Interessen an den für die Bevölkerung weniger salienten Themen arbeiten, dürfte die Themenrepräsentation der Bürgerinnen und Bürger durch diese Organisationen folglich gering sein.

Jedoch muss berücksichtigt werden, dass von den politischen Eliten ein Mindestmaß an Responsivität gegenüber gesellschaftlichen Bedürfnissen erwartet werden kann (Monroe 1998; Wlezien 1995, 2004). So haben Stimson et al. (1995) für die USA gezeigt, dass alle gewählten Institutionen, und in einem geringeren Maße auch der Supreme Court, auf Veränderungen der öffentlichen Meinung reagieren. Schließlich müssen auch nicht gewählte Akteure wie Bürokratinnen und Bürokraten, Richterinnen und Richter sowie Lobbyistinnen und Lobbyisten den Einfluss zukünftiger Wahlen auf das Verhalten der politischen Entscheider antizipieren, weil so der Raum politisch umsetzbarer Präferenzen bestimmt wird (Stimson et al. 1995, S. 545). Die Resonanz, die ihre eigenen Präferenzen und Handlungen im politischen Raum finden, wird entscheidend von der öffentlichen Meinung beeinflusst (Key 1962). Diese Rückbindung von politischen Akteuren an die Wählerschaft muss somit auch von organisierten Interessen berücksichtigt werden.

Die Beziehung zwischen öffentlicher Meinung und organisierten Interessen ist auch im Zusammenhang des ,outside lobbying“, also der öffentlichkeitsorientierten Kampagnen organisierter Interessen, von Bedeutung. Hierzu formulierte Kollman prägnant: „Policy preferences and issue salience among ordinary people comprise public opinion, and a useful image is to think of public opinion as the landscape upon which interest groups and elected officials operate“ (Kollman 1998, S. 25). So sehen Interessengruppen nach außen gerichtete Lobbyingstrategien, etwa durch die Mobilisierung ihrer Mitgliedschaft, dann als erfolgversprechend an, wenn sie dabei auch auf die Unterstützung einer breiten Öffentlichkeit bauen können (Kollman 1998). Zudem haben Rasmussen et al. (2017) gezeigt, dass in Deutschland und anderen westeuropäischen Ländern organisierte Interessen mit ihrem Lobbying erfolgreicher sind, wenn die Öffentlichkeit ähnliche Präferenzen hat. Auch im Kontext der EU-Gesetzgebung sind Interessengruppen und Politikerinnen und Politiker am responsivsten gegenüber den Forderungen der Öffentlichkeit, wenn die Themen salient sind (Rauh 2016). Deshalb erwarten wir, dass Bürgerinnen und Bürger, denen öffentlich saliente Themen wichtig sind, in einem stärkeren Maße von organisierten Interessen repräsentiert werden, als Bürgerinnen und Bürger, denen die weniger salienten Themen wichtig sind: 
H1 Je öffentlich-salienter ein Thema ist, das eine Bürgerin oder ein Bürger priorisiert, umso besser wird dieses Thema durch organisierte Interessen repräsentiert.

Jüngere Studien haben gezeigt, dass verschiedene Gruppen in der Bevölkerung unterschiedlich gut im politischen System repräsentiert werden. Giger et al. (2012) demonstrieren, dass vor allem einkommensstarke Bürgerinnen und Bürger eine ideologische Nähe zu den Regierungsparteien aufweisen. Elsässer et al. (2017) zeigen, dass die Unterschiede zwischen der Gesetzgebung und den Präferenzen der Bürgerinnen und Bürgern bei einkommensschwächeren Bevölkerungsschichten am größten sind. Beide Studien kommen somit zu dem Schluss, dass die Diskrepanzen in der Repräsentation durch Parteien vor allem zum Nachteil einkommensschwächerer Bevölkerungsgruppen gehen. Dies deckt sich außerdem mit dem Befund von Gilens und Page (2014). Sie haben herausgefunden, dass die Politikergebnisse stärker von den einkommensstärksten Bürgerinnen und Bürgern sowie Unternehmerinteressen bestimmt werden als von den Durchschnittsverdienerinnen und -verdienern in der Bevölkerung.

Vor diesem Hintergrund stellt sich die Frage, ob organisierte Interessen Ungleichheiten in der Repräsentation der Bürgerinnen und Bürger verstärken oder stattdessen zu einer besseren Repräsentation der Einkommensschwachen beitragen können. Die Forschung ist sich weitgehend einig, dass einkommensstärkere und gebildetere Teile der Bevölkerung besser in der Lage sind, sich politisch zu organisieren (Verba et al. 1995; Olson 1965; Schattschneider 1960). Denn individuelle Ressourcen wie das Einkommen sowie kommunikative und organisatorische Fähigkeiten sind einem zivilgesellschaftlichen Engagement sehr förderlich (Brady et al. 1995). So verfügen Bürgerinnen und Bürger, die Mitglieder in Verbänden sind oder sozialen Bewegungen angehören, nicht nur über ein höheres durchschnittliches Bildungsniveau, sondern auch über größere materielle Ressourcen (Verba et al. 1995). Dem liegt einerseits ein Selbstselektionsmechanismus, vermittelt über das politische Interesse, zugrunde. Andererseits fragen Interessengruppen diese persönlichen Ressourcen aktiv nach (Brady et al. 1995). So können Bürgerinnen und Bürger mit einem höheren soziökonomischen Status mehr Geld spenden und sich häufig auch stärker in die Arbeit der Organisationen einbringen (McCarthy und Zald 1977). Durch die Abhängigkeit der organisierten Interessen von ihren Geldgeberinnen und Geldgebern in vielen Fällen den Mitgliedern - sollten sich deren Interessen schließlich auch im Handeln der Interessengruppen wiederfinden (Lowery 2007). Daher kann angenommen werden, dass auch die Prioritäten der organisierten Interessen zugunsten der Präferenzen einkommensstärkere Menschen verzerrt sind, was zu unserer zweiten Hypothese führt:

H2 Je einkommensstärker eine Bürgerin oder ein Bürger ist, umso besser wird das von ihr oder ihm priorisierte Thema durch organisierte Interessen repräsentiert.

Bürgerinnen und Bürger, die sich auf der soziökonomischen Dimension links verorten, gleichzeitig jedoch traditionelle und autoritäre Wertvorstellungen haben, finden in westeuropäischen Parteiensystemen nur ein eingeschränktes Angebot (Boräng et al. 2018; Lefkofridi et al. 2014; Van der Brug und Van Spanje 2009). Auf- 
grund der Offenheit des Interessengruppensystems für Nischenorganisationen und -positionen können sich organisierte Interessen hingegen abseits der dominanten gesellschaftlichen und parteipolitischen Konfliktlinien positionieren. Dies ist nicht zuletzt der Logik des Entstehens und organisationalen Überlebens von Interessengruppen geschuldet (Lowery 2007; Wilson 1995). Interessengruppen vertreten in der Gesellschaft spezifische politische Themen und Präferenzen. Diese Angebote, die sie ihren Mitgliedern in ideologischer Sicht machen, sind mindestens so entscheidend wie die selektiven Anreize, mit denen Individuen dazu motiviert werden sollten, einer Interessengruppe beizutreten bzw. sie anderweitig zu unterstützen (Franke und Dobson 1985). Somit suchen sich Interessengruppen Policynischen, um sich in ihrer Positionierung voneinander abzugrenzen (Gray und Lowery 1996). Auch für ideologisch extremere Positionen finden sich Nischen, in denen einzelne Gruppen versuchen, einen Alleinvertretungsanspruch geltend zu machen (Lowery 2007). Eine der größten Gefahren für den Erfolg bzw. Fortbestand einer Organisation geht von ihr ideologisch nahestehenden Konkurrentinnen aus, also z. B. für Umweltorganisationen von anderen Organisationen, die in diesem Themenfeld engagiert sind. Entsprechende Strategien des Umgangs mit Konkurrenz bestehen in der Ausdifferenzierung, einschließlich der Radikalisierung, von Positionen (Lowery 2007: S. 42-43). So lassen sich bei Mitgliedern von Interessengruppen im Schnitt auch extremere ideologische Positionen vorfinden als bei Bürgerinnen und Bürgern, die nicht Mitglied in einer entsprechenden Organisation sind (Claassen und Nicholson 2013; Sabatier und McLaughlin 1990). Daher können auch unorganisierte Wählerinnen und Wähler mit spezifischen Policyinteressen durch gut organisierte und strategisch handelnde Interessengruppen repräsentiert werden. Die Wahrscheinlichkeit, dass sich Bürgerinnen und Bürger in diesen Nischen wiederfinden und somit von organisierten Interessen repräsentiert werden, sollte größer sein als im System der Repräsentation durch politische Parteien. So hat Romeijn (2018) herausgefunden, dass sich Nischen- und Mainstreamparteien in Deutschland nicht wesentlich in dem Grad unterscheiden, in dem sie sich an den Policypräferenzen der breiteren Öffentlichkeit orientieren. ${ }^{1}$ Hingegen können organisierte Interessen, die nicht um Wählerstimmen werben müssen, in ihrer Gesamtheit die ganze Bandbreite des Spektrums ideologischer Einstellungen in der Bevölkerung abdecken (Boräng et al. 2018). Daher erwarten wir, dass die „Außenseiter“ in der Wählerschaft im breiteren Angebot der unterschiedlichen organisierten Interessen fündig werden:

H3 Je extremer eine Bürgerin oder ein Bürger ideologisch positioniert ist, umso besser wird das von ihr oder ihm priorisierte Thema durch organisierte Interessen repräsentiert.

Auf der Ebene der organisierten Interessen erwarten wir, dass Unternehmen und Unternehmerverbände weniger repräsentativ für die Öffentlichkeit sind als andere Gruppen, weil sie eine geringere Bandbreite an gesellschaftlichen Interessen wider-

\footnotetext{
${ }^{1}$ Die entscheidende Trennlinie verlaufe in dieser Hinsicht zwischen Regierungs- und Oppositionsparteien. Romeijn (2018) betrachtet in seinem Untersuchungszeitraum von 1998-2010 CDU/CSU, SPD und FDP als Mainstream-, Grüne und Linke hingegen als Nischenparteien.
} 
spiegeln. Unter Unternehmerinteressen verstehen wir sämtliche Arbeitgeber- und Wirtschaftsverbände sowie einzelne Unternehmen (Baroni et al. 2014; siehe auch Baumgartner et al. 2009). Die meisten Unternehmerorganisationen haben mit Unternehmen oder spezialisierten Verbänden andere Organisationen als Mitglieder. Im Gegensatz dazu stehen Nichtunternehmerinteressen häufiger in direktem Kontakt mit ihren individuellen Mitgliedern, die wiederum zu einem großen Teil Wählerinnen und Wähler sind (Berry und Wilcox 2018). Gruppen, die auf individuelle Mitglieder abzielen, haben außerdem einen größeren Anreiz, sich politisch und gesellschaftlich salienter Themen anzunehmen (Lowery 2007). Und schließlich hat die Forschung gezeigt, dass Nichtunternehmerinteressen eher dazu neigen, neue Themen auf die politische Agenda zu bringen und sich für einen Politikwechsel einzusetzen, während Unternehmerinteressen eher den regulativen Status quo verteidigen (Dür et al. 2015). Das macht Nichtunternehmerinteressen oftmals zu Verbündeten von Bürgerinnen und Bürgern, die einen Handlungsbedarf der Regierung in einem bestimmten Politikfeld sehen. Aus diesen Gründen werden wir in unserer Analyse zwischen Unternehmer- und Nichtunternehmerinteressen differenzieren.

\section{Daten}

Um die Agenden der organisierten Interessen in Deutschland abzubilden, haben wir zwischen März und September 2017 insgesamt 119 Interviews mit Lobbyistinnen und Lobbyisten von Unternehmer- und Nichtunternehmerinteressen geführt. ${ }^{2}$ Die Stichprobe setzt sich aus unterschiedlichen Teilstichproben zusammen. Der erste Teil der Organisationen wurde mittels zweier Onlineverzeichnisse erhoben, wohingegen der zweite Teil aus Dokumenten der Bundestagsverwaltung stammt. Um die Verzeichnisstichprobe der organisierten Interessen $\mathrm{zu}$ ziehen, haben wir zunächst die Onlineversion des OECKL-Verzeichnisses deutscher Organisationen verwendet. ${ }^{3}$ Gemäß den Auswahlkriterien mussten die Organisationen politische Interessen vertreten und ein Mindestmaß an politischer Aktivität aufweisen (Berkhout et al. 2017). Darüber hinaus haben wir eine Zufallsstichprobe von Firmen aus dem Verzeichnis der 500 größten deutschen Firmen gezogen, das von der Tageszeitung Die Welt zusammengestellt wird (Stand: Januar 2017). Damit tragen wir der gestiegenen Rolle des Firmenlobbyings Rechnung und haben gleichzeitig eine ausreichende Varianz bei den Firmen sichergestellt, um Zufallsschwankungen zu verringern. Dieses Vorgehen ist in der einschlägigen Literatur gängige Praxis, da Firmen erst ab einer gewissen Größe regelmäßig selbst lobbyieren (Bernhagen und Mitchell 2009; Drope und Hansen 2006; Grier et al. 1994). Während die Verzeichnisstichprobe repräsentativer für die organisierten Interessen in Deutschland ist, besteht der zweite Teil aus den Interessengruppen und Unternehmen, die politisch besonders aktiv und relevant für die politischen Entscheidungsträgerinnen und -träger in Berlin sind. Diese Insiderstichprobe setzt sich aus den Unternehmen und Verbänden zusammen, die

\footnotetext{
2 Die Daten wurden im Rahmen des internationalen Forschungsprojekts „Agendas and Interest Groups“ (agendasandinterestgroups.org) erhoben.

3 https://www.oeckl.de/oeckl-online/ (Stand: Februar 2017).
} 
Tab. 1 Anzahl der Unternehmer- und Nichtunternehmerinteressen in der Stichprobe

\begin{tabular}{lll}
\hline & Insiderstichprobe & Verzeichnisstichprobe \\
\hline Unternehmerinteressen (Firmen) & $28(11)$ & $35(11)$ \\
Nichtunternehmerinteressen & 28 & 28 \\
Gesamt & 56 & 63 \\
\hline
\end{tabular}

in den Jahren 2011 und 2012 insgesamt mindestens zweimal zu öffentlichen Anhörungen in Ausschüssen des Deutschen Bundestags eingeladen wurden. ${ }^{4}$ Aus dieser Gesamtheit haben wir schließlich die Stichprobe der politisch aktivsten Interessenorganisationen und Unternehmen gezogen. Tab. 1 vermittelt einen Überblick über die Teilstichproben der organisierten Interessen, mit denen wir Interviews geführt haben.

Die Experteninterviews mit den Lobbyistinnen und Lobbyisten, die diese Interessenorganisationen vertreten, dauerten in der Regel zwischen 45 und 60 min. Insgesamt konnten wir auf diese Weise 797 Themen ermitteln. Dazu haben wir unsere Interviewpartnerinnen und -partner zunächst gefragt, welches die wichtigsten politischen Themen sind, mit denen die Organisation sich in den letzten sechs Monaten am meisten beschäftigt hat. Zusätzlich haben wir auch nach Themen gefragt, die aktuell nicht auf der Tagesordnung der Organisation stehen. Dies betrifft erstens Themen, die zwar wichtig für die Kernziele der Organisation sind, denen sie aber im letzten halben Jahr nicht viel Zeit widmen konnte. Zweitens haben wir danach gefragt, welche Themen die Organisation gerne auf der Regierungsagenda sähe. Und drittens haben wir nach Themen gefragt, welche sie nach Möglichkeit von der Regierungsagenda fernhalten will. Während der Interviews wurde darauf geachtet, den Fokus auf der bundespolitischen Ebene zu halten und EU-Themen nur aufzunehmen, sofern diese bereits mit einem konkreten Handeln des deutschen Gesetzgebers, etwa bei der innerstaatlichen Umsetzung von EU-Recht, verbunden sind.

Um die Agenda der Bürgerinnen und Bürger abzubilden, wurde eine repräsentative CATI-Umfrage unter 1002 Bürgerinnen und Bürgern, die älter als 18 sind und in Deutschland leben, durchgeführt. Die Befragung erfolgte im Oktober 2017, also im Nachgang der damaligen Bundestagswahl. Aufgrund unseres Forschungsinteresses und unter Berücksichtigung methodologischer Kritik an der Frage nach dem „wichtigsten Problem“ (siehe Wlezien 2005), haben wir den Respondentinnen und Respondenten die folgende Frage gestellt:

Die Bundesregierung trifft in vielen verschiedenen Bereichen Entscheidungen, die das Land und die einzelnen Bürger betreffen. Was ist derzeit aus Ihrer Perspektive das wichtigste Thema, bei dem Sie sich eine Veränderung der aktuellen Regierungspolitik wünschen würden?

Anstatt nach einem „Problem“ zu fragen, von dem die Bürgerinnen und Bürger erwarten oder nicht erwarten mögen, dass sich die Regierung darum kümmern soll-

\footnotetext{
4 Für das Jahr 2011 konnten wir auf einen Datensatz von Eising und Spohr (2016) zurückgreifen. Diesen haben wir für das Jahr 2012 unter Anwendung der Erhebungsmethoden dieser Autoren erweitert.
} 
te, haben wir also gefragt, welches Thema sie auf der Tagesordnung der Regierung sehen möchten. Diese Fragestellung erlaubt uns die Daten aus der Bevölkerungsumfrage und den Experteninterviews miteinander in Beziehung zu setzen. Ferner haben wir offene Folgefragen verwendet, in denen wir die Befragten gebeten haben, detaillierter zu beschreiben, was genau sie mit diesem Thema meinen und welcher Art der Wandel in der aktuellen Regierungspolitik sein sollte, den sie sich hier wünschen. In einigen Fällen wurden die Angaben hierzu verwendet, um ambivalente Nennungen zur ersten Frage einzuordnen.

Um die Agenden von organisierten Interessen und Bürgerinnen und Bürgern miteinander zu vergleichen, verwenden wir die deutsche Version des Kodierschemas des Comparative Agendas Project (CAP) (Breunig und Guinaudeau 2019). Dieses erlaubt die Klassifizierung politischer Themen in 21 Hauptthemen (,major codes“), die wiederum in 220 Unterthemen (,minor codes“) unterteilt sind. Alle Themen, die von befragten Bürgerinnen und Bürgern und/oder organisierten Interessen genannt worden sind, wurden einem Unterthema zugewiesen. Bürgerinnen und Bürger und organisierte Interessen können innerhalb eines Politikfelds recht unterschiedliche Agenden haben. Dies wird am Beispiel des Hauptthemas Bürger-, Freiheits- und Minderheitsrechte deutlich. Hier haben organisierte Interessen ein breiteres Interesse an verschiedenen Unterthemen innerhalb des Hauptthemas, während die Bürgerinnen und Bürger ganz überwiegend die Flüchtlingspolitik (Unterthema Einwanderung, Flüchtlinge, Aussiedler und Einbürgerung) genannt haben (Abb. 1). Aufgrund

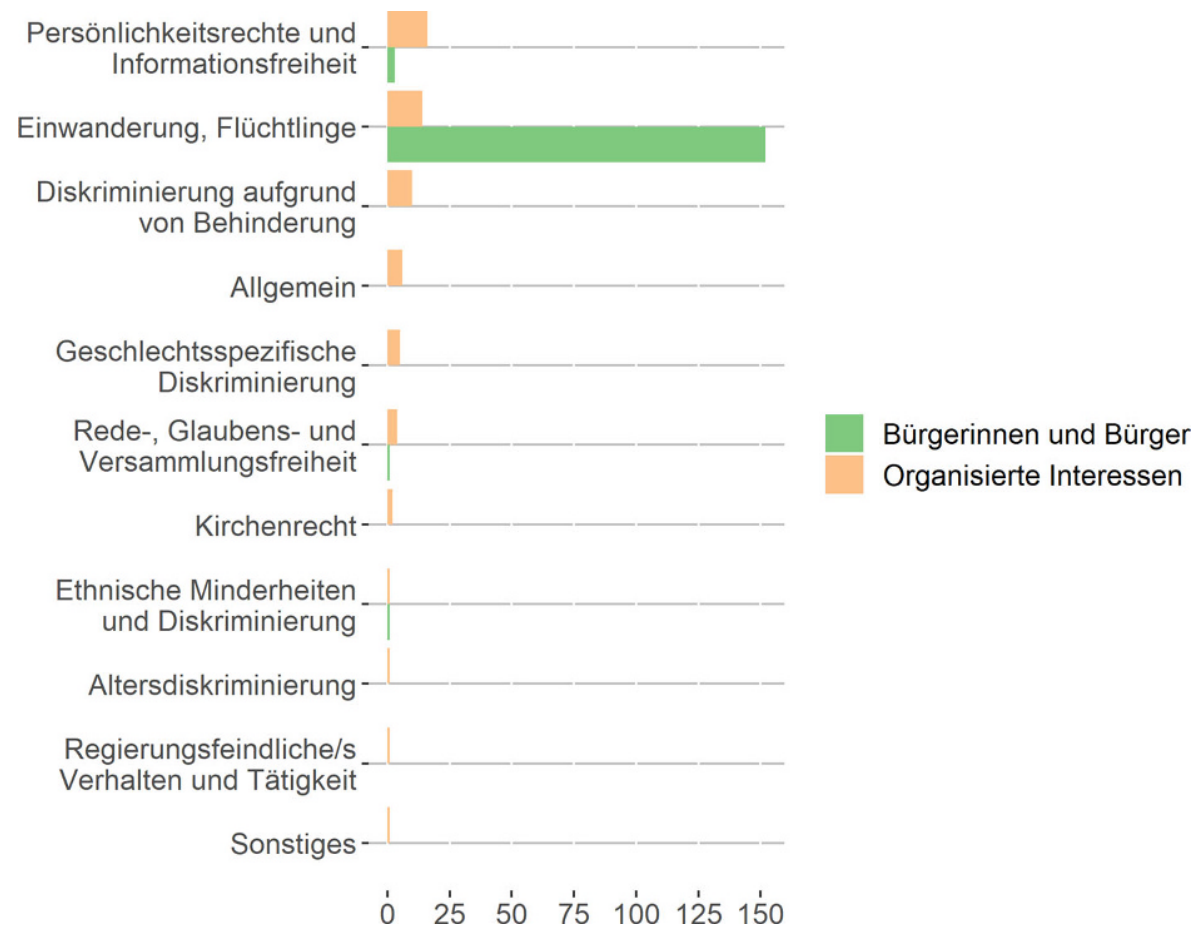

Abb. 1 Häufigkeit der Unterthemen im Hauptthema Bürger-, Freiheitrechte und Minderheitsrechte 
der großen Variation an Agenden innerhalb der Hauptthemen werden wir in der empirischen Analyse die Agenden von Bürgerinnen und Bürgern und organisierten Interessen auf der niedrigeren Aggregationsstufe, den Unterthemen (nachfolgend nur noch Themen genannt) miteinander vergleichen.

In beiden Teildatensätzen wurden die Kodierungen von mehreren trainierten Kodiererinnen und Koderiern durchgeführt. Gab es keine Mehrheitsmeinung, wurde von einem erfahrenem Forscher entschieden. Im Umfragedatensatz lagen solche uneindeutigen Kodierungen in 14,7\%, bei den Experteninterviews in 18,0\% der Fälle vor.

\section{Themenkongruenz zwischen Bürgerinnen und Bürgern und organisierten Interessen}

Zur besseren Verständlichkeit unseres Untersuchungsdesigns beginnen wir mit der Untersuchung der Kongruenz zwischen politischen Themen, welche die Bürgerinnen und Bürger auf der Regierungsagenda sehen möchten und jenen, an denen die organisierten Interessen arbeiten. Hier geht es darum, wie stark die Themen, die von Befragten genannt wurden, von organisierten Interessen aufgegriffen wurden. Die Untersuchungseinheit ist daher zunächst das (CAP-Unter-)Thema. Dabei betrachten wir die absoluten Häufigkeiten, wobei wir Themen, die weder von Bürgerinnen und Bürgern noch von organisierten Interessen genannt wurden, unberücksichtigt lassen. Nach Maßgabe der Häufigkeiten sind die Agenden moderat korreliert, in diesem Fall mit einem Pearsons $r$ von 0,37 (Abb. 2). ${ }^{5}$ Beschriftet sind in der Grafik die jeweils zehn von Befragten und organisierten Interessen am häufigsten genannten Themen. ${ }^{6}$ Auffällig ist dabei, dass die Themen Grundschule und weiterführende Schule sowie Bildung Allgemein bei der Bevölkerung häufig genannt werden. Wie in der Bevölkerungsumfrage haben wir in unseren Experteninterviews explizit nach Bundesthemen gefragt, konnten dabei jedoch die Exklusion von Länderthemen besser kontrollieren. Somit könnte die Unterrepräsentierung des Themas Bildung bei den organisierten Interessen ein Artefakt der Datenerhebung sein, das sich jedoch nicht maßgeblich auf die Ergebnisse auswirkt. ${ }^{7}$

In Abb. 3 sind sowohl die Agenden der organisierten Interessen als auch die der Bürgerinnen und Bürger nach Wichtigkeit sortiert und in einem Streudiagramm gegenübergestellt. Die Wichtigkeit entspricht dem umgekehrten Rang eines Themas,

\footnotetext{
5 Anders als Abb. 2 suggeriert, ist diese Korrelation nicht von einigen wenigen Ausreißern getragen. Die einschlägigen Diagnostikmaßzahlen wie Cook-Abstand und DFBETA sind unauffällig.

6 Aus Gründen der Darstellbarkeit wird bei Themen auf dem gleichen Rang für beide Agendengruppen jeweils nur eines beschriftet. Häufig ergibt sich dies daraus, dass es jeweils auch auf der anderen Agenda in der Top-10 steht. So werden als am dritthäufigsten (14-mal) genannte Themen bei den organisierten Interessen Einwanderung, Flüchtlinge, Aussiedler und Einbürgerung und Gesundheitswesen Allgemein beschriftet, weil sie auch bei den Bürgerinnen und Bürgern auf Platz 1 bzw. 10 stehen.

7 Insbesondere das Thema Schule betrifft den Bereich der Länderkompetenzen. Deshalb haben wir den Korrelationskoeffizienten nach Ausschluss des Themas Grundschule und weiterführende Schule (77 von insgesamt 200 Nennungen im Politikfeld Bildung) erneut berechnet. Der Wert steigt dann geringfügig auf 0,38 .
} 


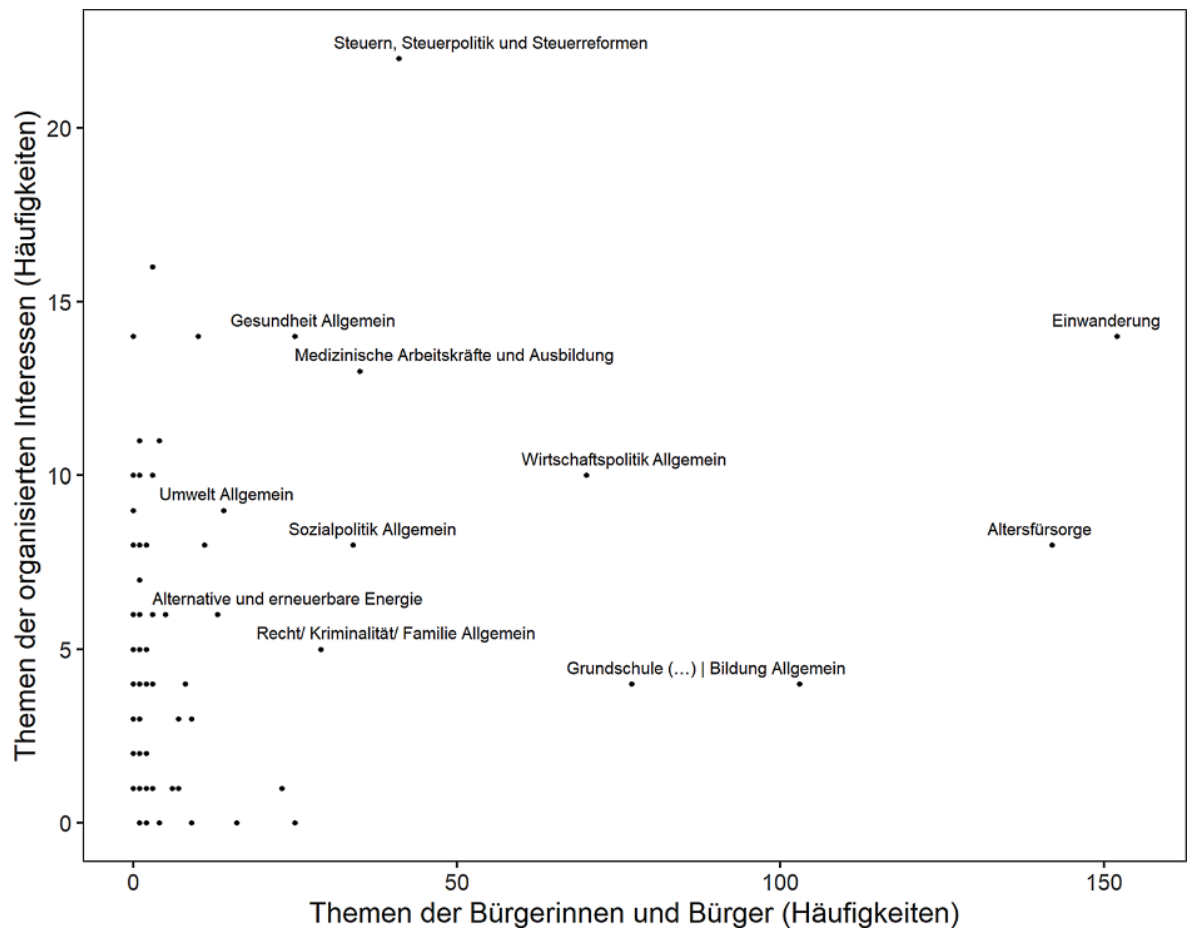

Abb. 2 Bivariater Zusammenhang zwischen den Themen der Bevölkerung und der organisierten Interes$\operatorname{sen}(n=159)$

was sich mit folgendem Beispiel veranschaulichen lässt: Die Immigration (Einwanderung, Flüchtlinge, Aussiedler und Einbürgerung) ist für die Bürgerinnen und Bürger das wichtigste Thema (Rang 1); ihm wird der Wert 80 zugewiesen, welcher dem Rang der am wenigsten für die Bürgerinnen und Bürger bedeutenden Politikfelder (z. B. Computer- und Informationssicherheit) entspricht, die jeweils einmal genannt wurden. Diese Themen wiederum haben die geringste Wichtigkeit (1). Dadurch werden die Werte entzerrt; ferner wird auf diese Weise dem Umstand Rechnung getragen, dass die Fallzahl der Bürgerinnen und Bürger deutlich höher ist als die Zahl der befragten organisierten Interessen. Vereinzelt kommt es vor, dass Themen - etwa Rente (Altersfürsorge) und Sozialpolitik Allgemein - dieselbe absolute Häufigkeit und damit auch dieselbe Wichtigkeit haben. Die meisten organisierten Interessen arbeiten an Themen aus dem Bereich Steuerpolitik und -reformen, denen somit hier die höchste Wichtigkeit zugewiesen wird. Die Korrelation zwischen den nach Rängen geordneten Themen ist Spearmans $\varrho=0,28 .{ }^{8}$ Während bei den Bürgerinnen und Bürgern außerdem die Themen Altersfürsorge und Bildung Allgemein ganz oben auf der Agenda stehen, arbeiten viele unserer befragten Organisationen an Themen der Digitalisierung (überwiegend kodiert als Persönlichkeitsrechte und Informationsfreiheit). Die Einwanderung steht bei den organisierten Interessen auf

\footnotetext{
8 Ohne das Thema Grundschule und weiterführende Schule: 0,27.
} 


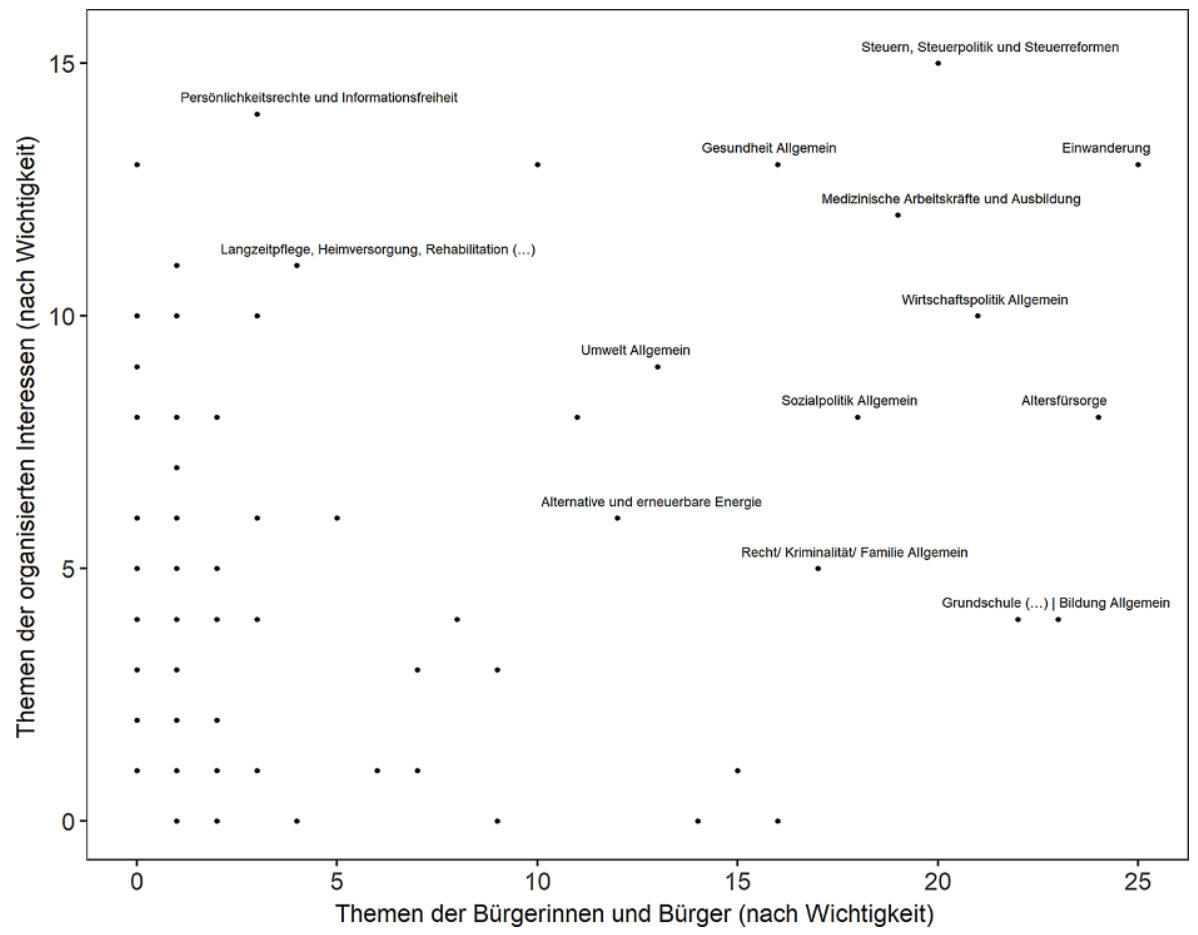

Abb. 3 Bivariater Zusammenhang zwischen nach Wichtigkeit geordneten Themen $(n=159)$

dem dritten Rang, gemeinsam mit Gesundheitswesen Allgemein, Luftverschmutzung, Klimaerwärmung und Lärmbelästigung sowie berufspolitischen Themen (kodiert als Arbeit Sonstiges).

Die berichteten Zusammenhangsmaße deuten auf eine mäßige Kongruenz zwischen den Agenden der Bevölkerung und der organisierten Interessen hin. Insoweit unterstützen sie Hypothese 1, wonach ein Thema umso mehr von organisierten Interessen bearbeitet wird, je wichtiger es den Bürgerinnen und Bürgern ist. Diese Erwartung wird auch durch die Schiefe der bivariaten Verteilung unterstützt: Die Themen, die eine niedrige Bedeutung für die Bürgerinnen und Bürger haben, sind recht gleichmäßig über das gesamte Spektrum von niedriger bis hoher Aufmerksamkeit durch die organisierten Interessen verteilt. Erst in Bereichen größerer Häufigkeit bzw. verstärkter Wichtigkeit in der Bevölkerung genießen die verbleibenden Themen zunehmend moderate bis hohe Vertretung durch Interessengruppen. Im Folgenden analysieren wir neben der Salienz eines Themas in der Bevölkerung die weiteren Faktoren, die auf einer individuellen Ebene den Grad der thematischen Repräsentation einer befragten Bürgerin oder eines Bürgers durch die organisierten Interessen beeinflussen. 


\section{Multivariate Analyse: Individuelle Themenkongruenz und Repräsentation durch organisierte Interessen}

Während die bivariate Analyse die Agenden von Bevölkerung und organisierten Interessen auf der Ebene der Themen abgeglichen hat, untersuchen wir nun, welche Faktoren gemäß unseren Hypothesen die thematische Repräsentation der einzelnen Bürgerin und des einzelnen Bürgers durch organisierte Interessen beeinflussen. Die abhängige Variable ist die Repräsentation der Bürgerinnenthemen durch organisierte Interessen. ${ }^{9}$ Um diese zu erfassen, addieren wir die Anzahl der organisierten Interessen, die an dem von einer Bürgerin oder einem Bürger genannten Thema arbeiten, und teilen die Summe durch die Gesamtzahl aller Themen, an denen mindestens eine Interessenorganisation in Deutschland arbeitet. ${ }^{10}$ Jeder und jedem Befragten wird somit der Wert als Prozentsatz zugewiesen, der ihrer individuellen thematischen Repräsentation durch organisierte Interessen hinsichtlich des Themas, bei dem sie sich eine Veränderung der aktuellen Regierungspolitik wünschen, entspricht. Von den insgesamt 550 Themen, an denen jeweils mindestens eine Organisation arbeitet, sind zum Beispiel 22 als Steuerpolitik und -reformen klassifiziert. Die individuelle thematische Repräsentation aller Bürgerinnen oder Bürger, welche sich eine Änderung der Regierungspolitik bei diesem Thema wünschen, beträgt in diesem Beispiel somit vier Prozent.

Die abhängige Variable Repräsentation der Bürgerinnenthemen durch organisierte Interessen hat eine Reichweite von 0 bis 4 . Das bedeutet, dass zwischen null und vier Prozent der Interessenorganisationen jeweils an dem Thema arbeiten, das die Bürgerin oder der Bürger in der Umfrage genannt hat. Bemessen an der Reichweite und den Maßen der zentralen Tendenz (arithmetisches Mittel=1,46; Median =1,45) deutet die Standardabweichung $(0,98)$ auf eine große themenbezogene Variation bei der Repräsentation hin, wobei die Daten keine besonderen Ausreißer aufweisen.

Da wir untersuchen wollen, ob sich die Auswirkungen von Salienz, Einkommen und ideologischer Verortung auf die Repräsentation der Bürgerinnenthemen nach bestimmten Typen von organisierten Interessen unterscheiden, teilen wir unsere Daten nach Organisationstyp auf, sodass im ersten Fall nur die Unternehmer- und im zweiten nur die Nichtunternehmerinteressen enthalten sind. Analog zum obigen Verfahren konstruieren wir somit zwei weitere abhängige Variablen: die Repräsentation der Bürgerinnenthemen durch Unternehmerinteressen und die Repräsentation der Bürgerinnenthemen durch Nichtunternehmerinteressen. Auf einer deskriptiven Ebene sehen wir, dass die Bürgerinnenthemen durch Nichtunternehmerinteressen (arithmetisches Mittel: 1,54; Median: 1,23) etwas besser vertreten werden als durch Unternehmerinteressen (arithmetisches Mittel: 1,39; Median: 0,98). Allerdings ist bei Letzteren die Spannbreite etwas größer. So reicht diese bei Unternehmerinter-

\footnotetext{
9 Zur Einfachheit der Darstellung verwenden wir hier nur die feminine Form („Bürgerinnenthemen“), es sind aber immer die Themen der Bürgerinnen und Bürger gemeint.

10 Wir können von Repräsentation durch organisierte Interessen sprechen, weil wir Themen, an denen eine Organisation mehrfach arbeitet, nur einmal zählen. So erhalten wir insgesamt 550 Themen, an denen jeweils mindestens eine Organisation arbeitet. Somit entspricht die Häufigkeit eines Unterthemas hier der Zahl der Organisationen, die an dem Thema arbeiten.
} 
essen von 0 bis $5,21 \%$, bei Nichtunternehmerinteressen variiert sie zwischen 0 und $3,7 \%$.

Die erste Haupterklärungsvariable, öffentliche Salienz, messen wir mit dem Prozentsatz, den ein Thema unter allen von den befragten Bürgerinnen und Bürgern genannten Themen erreicht. So hat zum Beispiel das Thema Einwanderung, Flüchtlinge, Aussiedler und Einbürgerung einen Wert von 15,6, was ausdrückt, dass fast jede(r) sechste Befragte dieses Thema als das wichtigste genannt hat, bei dem er oder sie sich eine Änderung in der Regierungspolitik wünscht. Somit wird jeder Befragten und jedem Befragten, die das Thema Einwanderung genannt haben, dieser Wert auf der Variablen öffentliche Salienz zugewiesen. Zur Messung unterschiedlicher Niveaus von Einkommen wurde eine ordinale Skala aus zehn Kategorien abgefragt, die von einem angegebenen monatlichen Einkommen von ,unter $500 €$ “ bis , $4500 €$ und mehr“ reicht. Um den ideologischen Extremismus zu ermitteln, haben wir zunächst die Links-Rechts-Ideologie mir einer 11-Punkte-Skala erfasst, die von Links (0) bis Rechts (10) reicht. ${ }^{11}$ Diese haben wir dann zu einer 6er-Skala „gefaltet“, wobei dem Skalenmittelwert (5) der Ideologieskala der Wert 0 auf der Extremismusvariable zugewiesen wird, während den Ideologiewerten 0 und 10 der Wert 5 zugewiesen wird.

Wir kontrollieren um weitere Faktoren, welche den Zusammenhang zwischen unseren Prädiktoren und der Repräsentation der Bürgerinnenthemen beeinflussen könnten. So schließen wir erstens die ideologische Selbsteinschätzung der Respondentinnen und Respondenten (Links-Rechts-Ideologie) in unsere Modelle ein. Zweitens haben wir die ,external political efficacy“ (politische Wirksamkeit) der Respondentinnen und Respondenten in die Regressionsmodelle aufgenommen. Diese wird mithilfe der Political-Efficacy-Kurzskala nach Beierlein et al. (2014) gemessen. ${ }^{12}$ Drittens haben wir die Parteiidentifikation der Befragten als Variable in die Modelle aufgenommen. Dabei konnten die Befragten zwischen allen seit der Bundestagswahl 2017 im Parlament vertretenen Parteien sowie den Optionen „Andere“ und „Keine“ auswählen. ${ }^{13}$ Demnach haben $36 \%$ der Befragten keinerlei Parteiidentifikation; diese Kategorie wurde von uns als Referenzgruppe verwendet. Viertens könnten sowohl das politische System als Ganzes als auch organisierte Interessen im Speziellen die Prioritäten der Bürgerinnen und Bürger in den alten Bundesländern besser abbilden als in den neuen. Denn zum einen wurden die meisten Interessengruppen im westlichen Teil des Landes sowie Berlins gegründet und haben auch heute dort noch ihren Sitz. Zum anderen ist der Organisationsgrad sowohl von Parteien als auch

\footnotetext{
11 Die entsprechende Frage lautet wie folgt: „,Viele Leute verwenden die Begriffe ,links “ und ,rechts ‘, wenn es darum geht, unterschiedliche politische Einstellungen zu kennzeichnen. Wenn Sie an Ihre eigenen politischen Ansichten denken, wo würden Sie diese auf einer Skala zwischen 0 und 10 einstufen? 0 bedeutet links, und 10 bedeutet rechts?".

12 Die entsprechende Frage lautet wie folgt: „Ich lese Ihnen nun einige Aussagen über Politiker vor. Bitte geben Sie jeweils an, ob Sie diesen Aussagen voll und ganz, eher, teils-teils, eher nicht oder überhaupt nicht zustimmen: ,Die Politiker kümmern sich darum, was einfache Leute denken“; ,die Politiker bemühen sich um einen engen Kontakt zur Bevölkerung."“.

13 Die Frage lautet wie folgt: „Viele Leute neigen längere Zeit einer bestimmten politischen Partei zu, obwohl sie auch ab und zu eine andere Partei wählen. Wie ist das bei Ihnen: Neigen Sie - ganz allgemein gesprochen - einer bestimmten Partei zu? Falls ja: Welcher?“.
} 
von Verbänden seit der Wiedervereinigung in den neuen Bundesländern erheblich niedriger als in den alten (von Beyme 2017). Die „external political efficacy“ ist in den neuen Bundesländern ebenfalls niedriger als im Westen (z. B. Thaidigsmann 2000). Aus diesen Gründen kontrollieren wir geograpfische Unterschiede, indem wir in der Analyse berücksichtigen, ob eine Befragte oder ein Befragter ihren Wohnsitz in einem der neuen Bundesländer hat (Neue Bundesländer=1, andernfalls 0). Schließlich werden Alter und Geschlecht als Kontrollvariablen in allen Regressionsmodellen berücksichtigt. Dabei ist das Alter eine kategoriale Variable, die aus sechs Kategorien (,18-24“ bis „65+“) besteht. Eine Zusammenfassung aller in den Regressionsanalysen verwendeten Variablen befindet sich in Tab. 2 im Anhang.

Wir schätzen zunächst ein lineares Regressionsmodell, um die Repräsentation der Bürgerinnenthemen durch organisierte Interessen zu prädizieren (Modell M1). ${ }^{14}$ In einem zweiten Schritt kodieren wir die abhängige Variable neu und analysieren einerseits die Repräsentation der Bürgerinnenthemen durch Unternehmerinteressen (M2a) und andererseits durch Nichtunternehmerinteressen (M2b). Da die Konstruktionen der abhängigen Variablen sowie der Variable öffentliche Salienz auf der Gruppierung der CAP-Unterthemen basiert, berechnen wir clusterrobuste Standardfehler. Um sicherzustellen, dass unsere Schätzungen weitgehend die soziodemografische Zusammensetzung der Bevölkerung widerspiegeln, gewichten wir die Bevölkerungsumfragedaten nach Geschlecht, Alter und Region anhand des letzten zum Zeitpunkt der Umfrage vorliegenden Mikrozensus des Statistischen Bundesamts.

Das Koeffizientendiagramm in Abb. 4 zeigt die Ergebnisse der multivariaten Regressionsanalysen mit Konfidenzintervallen auf 95\%-Konfidenzniveau. Zum Zweck der Übersichtlichkeit bilden wir nur die Effekte der metrischen Variablen ab; eine Regressionstabelle findet sich im Anhang (Tab. 3). ${ }^{15}$ Ein positives Vorzeichen der Steigungskoeffizienten zeigt einen positiven Zusammenhang zwischen der betreffenden Variable und der Repräsentation des Bürgerinnenthemas durch die (jeweiligen) organisierten Interessen an. Im ersten Modell mit allen Gruppen (M1) sind die Steigungskoeffizienten sowohl für die öffentliche Salienz als auch für den Extremismus signifikant. Beide weisen das nach Hypothesen 1 bzw. 3 erwartete positive Vorzeichen auf. Mit jedem Anstieg der öffentlichen Salienz eines Bürgerinnenthemas um einen Prozentpunkt erhöht sich die Repräsentation von Bürgerinnenthemen durch organisierte Interessen um 0,07 Prozentpunkte. Mit jedem Punktanstieg einer Bürgerin oder eines Bürgers auf der 6-Punkte-Extremismusskala, vergrößert sich der Anteil der organisierten Interessen, die an diesem Thema arbeiten, ebenfalls um 0,07 Prozentpunkte. Zwischen dem Einkommen der Bürgerinnen und Bürger und der Repräsentation ihrer Themen durch organisierte Interessen besteht hingegen,

\footnotetext{
14 Die Differenz aus den ursprünglich 1002 Befragten der Bevölkerungsumfrage und den 777 Beobachtungen in der Regressionstabelle (Tab. 3) im Anhang ergibt sich aus dem listenweisen Ausschluss von Fällen, die fehlende Werte aufweisen. So konnten 32 Befragten keine CAP-Unterthemen zugewiesen werden; die meisten fehlenden Werte auf individueller Ebene weisen die Variablen Parteiidentifikation sowie Einkommen auf (siehe Tab. 2 für die Fallzahlen der einzelnen Variablen).

15 Alle Regressionsmodelle wurden alternativ unter Ausschluss des Unterthemas Grundschule und weiterführende Schule berechnet; dabei gibt es keine substanziellen Abweichungen zu den Modellen unter Verwendung des vollständigen Datensatzes.
} 


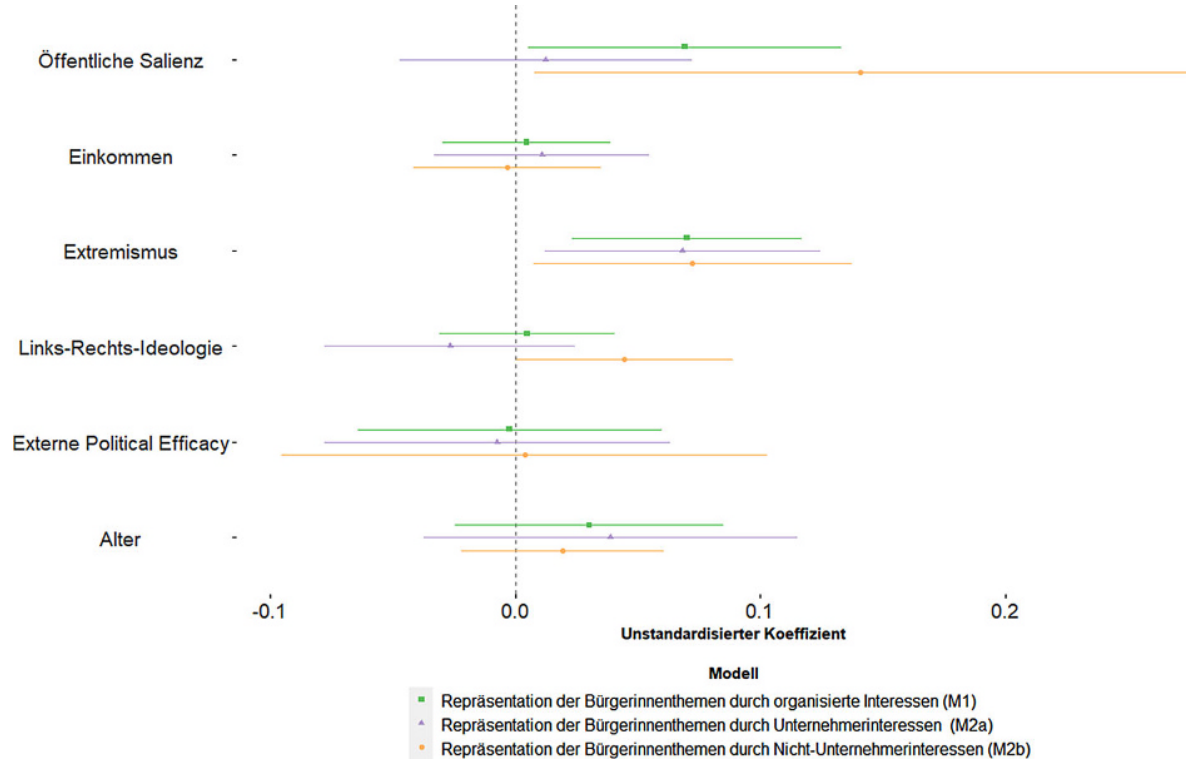

Abb. 4 Effekte der metrischen Prädiktoren der Themenkongruenz

anders als in Hypothese 2 erwartet, kein statistisch signifikanter Zusammenhang. Keine der Kontrollvariablen weist einen signifikanten Effekt auf.

Im Folgenden analysieren wir nun, ob es Unterschiede in der Repräsentationsleistung zwischen Unternehmer- und Nichtunternehmerorganisationen gibt. In diesen Modellen ist die Themenkongruenz nicht wie in M1 über alle organisierten Interessen hinweg erfasst, sondern nach Gruppentyp getrennt. Dabei fallen relevante Unterschiede hinsichtlich des Effekts der Variable öffentliche Salienz auf. Während dieser im Modell mit den Nichtunternehmerinteressen (M2b) etwa doppelt so stark ist wie im Grundmodell, ist der Koeffizient im Modell mit den Unternehmerinteressen (M2a) deutlich kleiner und nicht mehr signifikant. Somit tragen vor allem die Nichtunternehmerinteressen zum positiven Effekt der öffentlichen Salienz auf die Vertretung der Bürgerinnenthemen durch organisierte Interessen bei. Sowohl die Signifikanz als auch die Stärke des Effekts von Extremismus ändern sich allerdings nicht nennenswert in den beiden getrennten Modellen. Im Modell mit den Nichtunternehmerinteressen ist der Steigungskoeffizient für die ideologische Links-RechtsSelbstverortung signifikant und positiv. Dies bedeutet, dass Themen in einem höheren Maße von Nichtunternehmerinteressen vertreten werden, je weiter rechts die Bürgerinnen und Bürger sich politisch verorten, die diese Themen priorisieren.

Die übrigen Kontrollvariablen weisen auch in den getrennten Modellen keine signifikanten Effekte auf. Dies deutet darauf hin, dass sich die Themenrepräsentation der verschiedenen Bevölkerungsgruppen (z. B. Frauen und Ostdeutsche) oder hinsichtlich der politischen Einstellung sowie Parteiidentifikation zwischen den Unternehmer- und Nichtunternehmerinteressen jeweils nicht unterscheidet. Ebenso wenig bestehen Unterschiede bezüglich der ,external political efficacy“. 
Insgesamt ist die Vertretung der Bürgerinnenthemen durch die Nichtunternehmerinteressen stärker von der Salienz der Themen und den Einstellungen der Bürgerinnen und Bürgern geprägt als ihre Vertretung durch Unternehmerinteressen. Das R-Quadrat unterscheidet sich erheblich zwischen den drei Modellen: Während das Modell mit den Nichtunternehmerinteressen, das einen starken Effekt der öffentlichen Salienz aufweist, knapp $43 \%$ der Varianz aufklärt, sind es bei dem Modell mit den Unternehmerinteressen nicht einmal $2 \%$.

\section{Diskussion und Schlussfolgerungen}

Inwiefern können organisierte Interessen Defizite der Repräsentation durch das Parteiensystem kompensieren und gesellschaftliche Präferenzen in das politische System transportieren? Auf Basis der Literatur zu Repräsentation und organisierten Interessen haben wir die Erwartung formuliert, dass eine höhere Bedeutung eines Themas für die Bürgerinnen und Bürger mit einer stärkeren Berücksichtigung durch organisierte Interessen einhergeht. Ferner haben wir argumentiert, dass Bürgerinnen und Bürger umso besser thematisch durch Interessenorganisationen repräsentiert werden, je höher ihr Einkommen und je extremer ihre politischen Einstellungen sind. Die Ergebnisse unserer empirischen Analyse stützen unsere erste und dritte Hypothese. Zwar arbeiten organisierte Interessen hauptsächlich an ,ihren“ verbandsoder unternehmensspezifischen Themen, also jenen, die für ihre Mitglieder bzw. für ihre langfristigen Ziele als Organisation von Bedeutung sind. Der positive Einfluss der öffentlichen Salienz deutet jedoch darauf hin, dass organisierte Interessen die Themen der Bürgerinnen und Bürger in verstärktem Maße aufgreifen, wenn diese Themen für eine breitere Öffentlichkeit salient sind. In diesem Sinne findet eine Themenrepräsentation der Bürgerinnen und Bürger durch organisierte Interessen statt. Dieser Befund ist mit der Logik des Transmissionsriemens ebenso vereinbar wie mit dem Argument, dass organisierte Interessen auf Themen aufspringen, die in der Bevölkerung salient sind (Bandwagon-Effekt). Ferner deuten unsere Befunde an, dass organisierte Interessen die Themen von Bürgerinnen und Bürgern mit extremeren ideologischen Einstellungen besser repräsentieren als jene der ideologischen Mitte. Dieser Befund entspricht den populationsökologischen Erwartungen nach Gray und Lowery (1996) bezüglich der Nischenstrategien organisierter Interessen. Somit könnten organisierte Interessen eine Repräsentationslücke schließen, welche die Parteien, die sich an der Mitte des ideologischen Spektrums oder zumindest breiteren Wählergruppen orientieren müssen, offenlassen.

In Bezug auf Hypothese 1 müssen wir ferner hinsichtlich des Typs der Interessenorganisation differenzieren. So kommt der positive und signifikante Effekt im Grundmodell vor allem durch die Orientierung von Nichtunternehmerinteressen an den Themen zustande, die für die Bevölkerung bedeutend sind. Unternehmerinteressen scheinen in ihrer Arbeit hingegen von der öffentlichen Salienz weitgehend unbeeinflusst zu sein. Diese Befunde deuten darauf hin, dass organsierte Interessen nur in einem begrenzten Maße die Funktion übernehmen können, die Präferenzen der Bürgerinnen und Bürger in das politische System zu übertragen. So sind es in erster Linie zivilgesellschaftliche Organisationen, deren Arbeit mit der öffentlichen 
Salienz von bestimmten Themen korrespondiert, während Unternehmerinteressen eine Agenda verfolgen, die von solchen Überlegungen eher entkoppelt ist. Ein in der Literatur vermuteter Anspruch breiter aufgestellter Unternehmerinteressen, als Stimme größerer Bevölkerungsgruppen oder gar der Allgemeinheit zu fungieren (Bernhagen 2017, S. 45-47), wird durch diesen Befund in Zweifel gezogen.

Die Erwartung aus Hypothese 2, dass die Themen höherer Einkommensgruppen besser von Interessenorganisationen vertreten werden als die der Niedrigverdienerinnen und -verdiener findet keine Unterstützung in den Daten. Gemäß Hypothese 3 werden Bürgerinnen und Bürger, die extremere ideologische Einstellungen aufweisen, besser durch organisierte Interessen repräsentiert als jene mit moderaten Einstellungen. Dabei lassen sich keine nennenswerten Unterschiede hinsichtlich des Organisationstyps aufzeigen. Die Themen von Bürgerinnen und Bürgern mit extremeren ideologischen Einstellungen werden sowohl durch Unternehmerinteressen als auch durch Nichtunternehmerinteressen in einem höheren Maße vertreten als jene der ideologisch moderateren Bürgerinnen und Bürger. Dies könnte darauf hindeuten, dass die ideologischen „Außenseiter“ in der Wählerschaft durch das breitere Angebot der unterschiedlichen organisierten Interessen besser bedient werden als durch das Parteiensystem. In diesem Zusammenhang fällt jedoch abschließend auf, dass die Repräsentation von Bürgerinnenthemen durch Nichtunternehmerinteressen ansteigt, je weiter rechts sich die Bürgerinnen und Bürger ideologisch verorten. Eine mögliche Erklärung für dieses nicht unbedingt intuitive Ergebnis könnte in einer Limitation unserer quantitativen Analyse liegen, die keine Positionalität berücksichtigt. Wenn beispielsweise viele Befragte eine restriktivere Flüchtlingspolitik fordern, haben sie nach unserer Operationalisierung eine Repräsentation durch alle organisierten Interessen, die an diesem Thema arbeiten, auch wenn diese in der Frage in der Regel eine permissive Position einnehmen.

Haben wir mit unserem Fokus auf die Repräsentation von Themen also einen Nebenschauplatz der Transmissionsfunktion organisierter Interessen behandelt und damit womöglich eine Repräsentationsleistung gesehen wo es gar keine gibt? Wir denken, dass wir diese Frage verneinen können. Unsere Studie untersucht die thematische Repräsentation von Bürgerinnen und Bürgern durch organisierte Interessen im politischen System der BRD. Da ein Mindestmaß an Themenkongruenz eine Voraussetzung für das Funktionieren organisierter Interessen als Transmissionsriemen ist, sind die hier berichteten Ergebnisse von hoher demokratietheoretischer Relevanz. Themenkongruenz ist die Voraussetzung für einen politischen Diskurs und daraus resultierende Entscheidungsfindung (Dahl 1989). Unsere bivariate Analyse zeigt, dass die Themen, an denen die organisierten Interessen arbeiten, lediglich in einem moderaten Maße mit den Themen, die ganz oben auf der Tagesordnung der Bürgerinnen und Bürger stehen, übereinstimmen. Dieser Befund könnte zu eher pessimistischen Schlussfolgerungen hinsichtlich der Frage verleiten, inwiefern organisierte Interessen als Transmissionsriemen zwischen Bürgerinnen und Bürgern und politischen Eliten fungieren können. Allerdings ist die hier berichtete Themenkongruenz zwischen Bevölkerung und organisierten Interessen vor dem Hintergrund der Befunde von Kimball et al. (2012) zu sehen, welche für die USA keinen Zusammenhang zwischen öffentlicher und Interessengruppenagenda nachweisen konnten. Darüber hinaus haben wir anhand der multivariaten Analyse gesehen, dass zumindest Nicht- 
unternehmerinteressen zur Repräsentation von Bürgerinnenthemen beitragen. Somit leistet dieser Teil der Lobbylandschaft einen positiven Beitrag zur Repräsentativität des politischen Systems. Und schließlich können sowohl Unternehmerinteressen als auch Nichtunternehmerinteressen die thematische Repräsentation von Bürgerinnen und Bürgern mit extremeren ideologischen Einstellungen verbessern. Allerdings bleibt festzuhalten, dass Unternehmerinteressen insgesamt in einem deutlich geringeren Maße zu einer Repräsentation gesellschaftlicher Interessen beitragen können, als Nichtunternehmerinteressen dies vermögen.

Abschließend sei darauf hingewiesen, dass die neutralen Befunde zu den soziodemografischen sowie einigen Einstellungsvariablen demokratietheoretisch begrüßenswert sind: Bei den Themen, an denen die organisierten Interessen arbeiten, scheinen sie in ihrer Gesamtheit gegenüber sozioökonomischen Unterschieden blind zu sein. So werden die wichtigsten Themen der Bürgerinnen und Bürger mit geringeren Einkommen, der Frauen, Jüngeren und Ostdeutschen weder besser noch schlechter durch die organisierten Interessen vertreten als die der jeweils anderen gesellschaftlichen Gruppen. Gleiches gilt für die Anhängerinnen und Anhänger von bestimmten politischen Parteien sowie diejenigen, die sich mit keiner politischen Partei identifizieren oder sich vom politischen System nicht gehört fühlen (geringe „external political efficacy“).

Mit Deutschland als korporatistischer Verhandlungsdemokratie und einem Mehrparteiensystem wirft diese Untersuchung erstmals ein Schlaglicht auf eine größere Gruppe von Untersuchungsfällen, die in der bisherigen Forschung vernachlässigt worden sind. Unser neutraler Befund hinsichtlich eines Einkommenseffekts auf die Themenrepräsentation stimmt dabei mit der Vermutung von Giger et al. (2012) überein, dass die Präferenzen der einkommensstärkeren Bevölkerungsschichten in Ländern mit Mehrheitswahlsystemen stärker überrepräsentiert werden als in Ländern mit Verhältniswahlsystemen. Allerdings lässt unsere Untersuchung keinen Rückschluss auf die Ergebnisse des politischen Prozesses zu, etwa in Form verabschiedeter Gesetze.

Abschließend sei auf zwei Limitationen unserer Studie hingewiesen. Erstens haben wir, wie bereits erwähnt, zu den erfassten Themen keine Positionen von Bürgerinnen und Bürgern oder organisierten Interessen untersucht. Daher ist es auch bei den Themen mit geteilter Salienz möglich, dass wir das repräsentative Potenzial organisierter Interessen überschätzen. Tatsächlich können Organisationen, die an einem Thema arbeiten und Bürgerinnen und Bürger, die dieses wichtig finden, grundlegend unterschiedliche Positionen haben. Hier sei noch einmal auf das erwähnte Beispiel der Flüchtlingspolitik verwiesen. Somit beziehen sich unsere Schlussfolgerungen allein auf die Themenkongruenz zwischen organisierten Interessen und Bürgerinnen und Bürgern. Zweitens können wir die zeitliche Abfolge der Thematisierungen durch Bevölkerung und organisierte Interessen nicht kontrollieren. Theoretisch könnte der Wirkungszusammenhang daher auch in die andere Richtung laufen, indem organisierte Interessen die Bevölkerung für bestimmte Themen sensibilisieren. Insofern handelt es sich bei unserer Untersuchung nicht um einen kritischen Test, da die Daten zwar die Transmissionsriemenlogik des Repräsentationspotenzials organisierter Interessen stützen, alternative Erklärungen der nachgewiesenen Themenkongruenz jedoch nicht widerlegt werden. 
Für das grundlegende Kriterium der Demokratie, wonach die Bürgerinnen und Bürger Einfluss auf die politische Agenda haben sollen (Dahl 1989), muss zuallererst vorausgesetzt werden, dass sich die relevanten Akteure mit den gleichen Themen beschäftigen können. Unsere Arbeit liefert somit einen Erkenntnisgewinn hinsichtlich der Aggregationslogiken in der intermediären Sphäre der Politik. So konnten wir erstens zeigen, dass es eine moderate Kongruenz zwischen Bürgerinnenthemen und den Betätigungsfeldern organisierter Interessen gibt. Zweitens lässt sich das Ausmaß der Vertretung der Bürgerinnenthemen auf der Individualebene durch ideologisch extreme Einstellungen vorhersagen. Beide Repräsentationsleistungen werden von Nichtunternehmerinteressen in stärkerem Maße erbracht als von Unternehmerinteressen. Dies unterstreicht die Bedeutung von organisierten Interessen, insbesondere den Nichtunternehmerinteressen, als intermediäre Organisationen. Im politischen System der Bundesrepublik aggregieren diese Organisationen gesellschaftliche Interessen und bearbeiten auch die Themen derjenigen Bürgerinnen und Bürger, die sich abseits der politischen Mitte befinden.

Danksagung Wir danken Jeroen Romeijn und zwei anonymen GutachterInnen für hilfreiche Kommentare sowie Johannes Baamann, Tom Huhnke, Katrin Jochum und Tamara Schnell für ihre Unterstützung bei der Datenkodierung.

Gefördert durch die Deutsche Forschungsgemeinschaft (DFG) - Projektnummer BE 3973/1-1.

Funding Open Access funding provided by Projekt DEAL.

Open Access Dieser Artikel wird unter der Creative Commons Namensnennung 4.0 International Lizenz veröffentlicht, welche die Nutzung, Vervielfältigung, Bearbeitung, Verbreitung und Wiedergabe in jeglichem Medium und Format erlaubt, sofern Sie den/die ursprünglichen Autor(en) und die Quelle ordnungsgemäß nennen, einen Link zur Creative Commons Lizenz beifügen und angeben, ob Änderungen vorgenommen wurden.

Die in diesem Artikel enthaltenen Bilder und sonstiges Drittmaterial unterliegen ebenfalls der genannten Creative Commons Lizenz, sofern sich aus der Abbildungslegende nichts anderes ergibt. Sofern das betreffende Material nicht unter der genannten Creative Commons Lizenz steht und die betreffende Handlung nicht nach gesetzlichen Vorschriften erlaubt ist, ist für die oben aufgeführten Weiterverwendungen des Materials die Einwilligung des jeweiligen Rechteinhabers einzuholen.

Weitere Details zur Lizenz entnehmen Sie bitte der Lizenzinformation auf http://creativecommons.org/ licenses/by/4.0/deed.de. 


\section{Anhang}

Tab. 2 Deskriptive Statistiken der in den Regressionen verwendeten Variablen

\begin{tabular}{|c|c|c|c|c|c|c|}
\hline & $N$ & $\begin{array}{l}\text { Arithmeti- } \\
\text { sches } \\
\text { Mittel }\end{array}$ & $\begin{array}{l}\text { Standard- } \\
\text { abweichung }\end{array}$ & Minimum & Median & Maximum \\
\hline $\begin{array}{l}\text { Repräsentation der Bür- } \\
\text { gerinnenthemen durch } \\
\text { organisierte Interessen }\end{array}$ & 970 & 1,46 & 0,03 & 0 & 1,45 & 4 \\
\hline $\begin{array}{l}\text { Repräsentation der Bür- } \\
\text { gerinnenthemen durch } \\
\text { Unternehmerinteressen }\end{array}$ & 970 & 1,39 & 1,12 & 0 & 0,98 & 5,21 \\
\hline $\begin{array}{l}\text { Repräsentation der Bür- } \\
\text { gerinnenthemen durch } \\
\text { Nichtunternehmerinteressen }\end{array}$ & 970 & 1,54 & 1,22 & 0 & 1,23 & 3,70 \\
\hline Öffentliche Salienz & 970 & 7,75 & 0,18 & 0,10 & 7,22 & 15,67 \\
\hline Einkommen & 864 & - & - & 1 & 6 & 10 \\
\hline Links-Rechts-Ideologie & 984 & - & - & 0 & 4 & 10 \\
\hline Extremismus & 984 & - & - & 0 & 1 & 5 \\
\hline „External political efficacy“ & 996 & 3,75 & 0,03 & 1 & 4 & 5 \\
\hline Neue Bundesländer & 1002 & 0,22 & - & 0 & 0 & 1 \\
\hline Weiblich & 1002 & 0,46 & - & 0 & 0 & 1 \\
\hline Alter & 1002 & - & - & 1 & 4 & 6 \\
\hline \multicolumn{7}{|l|}{ Parteiidentifikation } \\
\hline $\mathrm{CDU} / \mathrm{CSU}$ & 929 & 0,23 & - & 0 & 0 & 1 \\
\hline SPD & 929 & 0,18 & - & 0 & 0 & 1 \\
\hline FDP & 929 & 0,05 & - & 0 & 0 & 1 \\
\hline Die Grünen & 929 & 0,08 & - & 0 & 0 & 1 \\
\hline Die Linke & 929 & 0,07 & - & 0 & 0 & 1 \\
\hline AfD & 929 & 0,03 & - & 0 & 0 & 1 \\
\hline Andere Partei & 929 & 0,02 & - & 0 & 0 & 1 \\
\hline
\end{tabular}


Tab. 3 Multivariate Modelle (OLS-Regression)

\begin{tabular}{|c|c|c|c|}
\hline $\begin{array}{l}\text { Abhängige } \\
\text { Variable }\end{array}$ & $\begin{array}{l}\text { M1 } \\
\text { Repräsentation durch } \\
\text { organisierte Interessen }\end{array}$ & $\begin{array}{l}\text { M2a } \\
\text { Repräsentation durch } \\
\text { Unternehmerinteressen }\end{array}$ & $\begin{array}{l}\text { M2b } \\
\text { Repräsentation durch } \\
\text { Nichtunternehmerinter- } \\
\text { essen }\end{array}$ \\
\hline $\begin{array}{l}\text { Öffentliche } \\
\text { Salienz }\end{array}$ & $\begin{array}{l}0,069 * \\
(0,033)\end{array}$ & $\begin{array}{l}0,012 \\
(0,030)\end{array}$ & $\begin{array}{l}0,141^{* *} \\
(0,068)\end{array}$ \\
\hline Einkommen & $\begin{array}{l}0,005 \\
(0,018)\end{array}$ & $\begin{array}{l}0,011 \\
(0,022)\end{array}$ & $\begin{array}{c}-0,003 \\
(0,020)\end{array}$ \\
\hline $\begin{array}{l}\text { Links- } \\
\text { Rechts- } \\
\text { Ideologie }\end{array}$ & $\begin{array}{l}0,005 \\
(0,018)\end{array}$ & $\begin{array}{l}-0,027 \\
(0,026)\end{array}$ & $\begin{array}{l}0,044 * \\
(0,023)\end{array}$ \\
\hline Extremismus & $\begin{array}{l}0,070^{* *} \\
(0,024)\end{array}$ & $\begin{array}{l}0,068^{*} \\
(0,029)\end{array}$ & $\begin{array}{l}0,072^{*} \\
(0,033)\end{array}$ \\
\hline $\begin{array}{l}\text { „External } \\
\text { political } \\
\text { efficacy“ }\end{array}$ & $\begin{array}{l}-0,002 \\
(0,032)\end{array}$ & $\begin{array}{l}-0,007 \\
(0,036)\end{array}$ & $\begin{array}{l}0,004 \\
(0,051)\end{array}$ \\
\hline $\begin{array}{l}\text { Neue Bun- } \\
\text { desländer }\end{array}$ & $\begin{array}{l}-0,041 \\
(0,056)\end{array}$ & $\begin{array}{l}-0,023 \\
(0,067)\end{array}$ & $\begin{array}{l}-0,063 \\
(0,082)\end{array}$ \\
\hline Weiblich & $\begin{array}{l}-0,002 \\
(0,093)\end{array}$ & $\begin{array}{l}-0,025 \\
(0,116)\end{array}$ & $\begin{array}{l}0,027 \\
(0,115)\end{array}$ \\
\hline Alter & $\begin{array}{l}0,030 \\
(0,028)\end{array}$ & $\begin{array}{l}0,039 \\
(0,039)\end{array}$ & $\begin{array}{l}0,019 \\
(0,021)\end{array}$ \\
\hline \multicolumn{4}{|c|}{ Parteiidentifikation (Referenzkategorie: „keine“) } \\
\hline $\mathrm{CDU} / \mathrm{CSU}$ & $\begin{array}{l}-0,011 \\
(0,066)\end{array}$ & $\begin{array}{l}0,009 \\
(0,077)\end{array}$ & $\begin{array}{l}-0,036 \\
(0,088)\end{array}$ \\
\hline SPD & $\begin{array}{l}-0,140 \\
(0,110)\end{array}$ & $\begin{array}{l}-0,092 \\
(0,155)\end{array}$ & $\begin{array}{l}-0,201 \\
(0,115)\end{array}$ \\
\hline FDP & $\begin{array}{l}0,351 \\
(0,203)\end{array}$ & $\begin{array}{l}0,489 \\
(0,297)\end{array}$ & $\begin{array}{l}0,177 \\
(0,125)\end{array}$ \\
\hline Die Grünen & $\begin{array}{l}-0,009 \\
(0,201)\end{array}$ & $\begin{array}{l}0,158 \\
(0,224)\end{array}$ & $\begin{array}{l}-0,220 \\
(0,265)\end{array}$ \\
\hline Die Linke & $\begin{array}{l}-0,073 \\
(0,148)\end{array}$ & $\begin{array}{l}0,082 \\
(0,199)\end{array}$ & $\begin{array}{l}-0,268 \\
(0,201)\end{array}$ \\
\hline AfD & $\begin{array}{l}-0,037 \\
(0,139)\end{array}$ & $\begin{array}{l}-0,074 \\
(0,207)\end{array}$ & $\begin{array}{l}0,009 \\
(0,145)\end{array}$ \\
\hline $\begin{array}{l}\text { Andere } \\
\text { Partei }\end{array}$ & $\begin{array}{l}0,238 \\
(0,210)\end{array}$ & $\begin{array}{l}0,354 \\
(0,302)\end{array}$ & $\begin{array}{l}0,093 \\
(0,190)\end{array}$ \\
\hline Konstante & $\begin{array}{l}0,600 \\
(0,331)\end{array}$ & $\begin{array}{l}0,955^{* * *} \\
(0,368)\end{array}$ & $\begin{array}{l}0,152 \\
(0,516)\end{array}$ \\
\hline$N$ & 777 & 777 & 777 \\
\hline $\begin{array}{l}\text { Bereinigtes } \\
R^{2}\end{array}$ & 0,174 & 0,018 & 0,425 \\
\hline$F$ & $11,892^{* * * *}$ & $1,966^{*}$ & $39,199^{* * *}$ \\
\hline
\end{tabular}

Clusterrobuste Standardfehler in Klammern $* p<0,05 ; * * p<0,01 ; * * * p<0,001$ 


\section{Literatur}

Agnone, Jon. 2007. Amplifying public opinion: the policy impact of the U.S. Environmental movement. Social Forces 85(4):1593-1620.

Baroni, Laura, Brendan J. Carroll, Adam W. Chalmers, Luz M.M. Marquez, und Anne Rasmussen. 2014. Defining and classifying interest groups. Interest Groups \& Advocacy 3(2):141-159.

Bartels, Larry M. 2016. Unequal democracy: the political economy of the new gilded age. Princeton: Princeton University Press.

Baumgartner, Frank R., und Beth L. Leech. 1998. Basic interests. The importance of groups in politics and in political science. Princeton: Princeton University Press.

Baumgartner, Frank R., und Beth L. Leech. 2001. Interest niches and policy bandwagons: patterns of interest group involvement in national politics. Journal of Politics 63(4):1191-1213.

Baumgartner, Frank R., Jeffrey M. Berry, Marie Hojnacki, David C. Kimball, und Beth L. Leech. 2009. Lobbying and policy change. Who wins, who loses, and why. Chicago: University of Chicago Press.

Beierlein, Constanze, Christoph J. Kemper, Anastassyia Kovaleva, und Beatrice Rammstedt. 2014. Political Efficacy Kurzskala (PEKS). Zusammenstellung sozialwissenschaftlicher Items und Skalen. https://zis.gesis.org/pdfFiles/Dokumentation/Beierlein\%2B_Political_Efficiacy_(PEKS)_c.pdf. Zugegriffen: 30. Dez. 2019.

Berkhout, Joost, Marcel Hanegraaff, und Caelesta Braun. 2017. Is the EU different? Comparing the diversity of national and EU-level systems of interest organisations. West European Politics 40(5):1109-1131.

Bernhagen, Patrick. 2017. Wirtschaftskammern als politische Akteure: Organisation, Strategie und Einfluss. In Wirtschaftskammern im europäischen Vergleich, Hrsg. Detlef Sack, 31-54. Wiesbaden: Springer VS.

Bernhagen, Patrick, und Neil J. Mitchell. 2009. The determinants of direct corporate lobbying in the European Union. European Union Politics 10(2):155-176.

Berry, Jeffrey M., und Clyde Wilcox. 2018. The interest group society. New York, London: Taylor \& Francis.

Bevan, Shaun, und Anne Rasmussen. 2017. When does government listen to the public? Voluntary associations and dynamic agenda representation in the United States. Policy Studies Journal 48(1):1-22.

Boräng, Frida, Daniel Naurin, und Jonathan Polk. 2018. Making space. A cross-country comparison of party and interest group positioning in multiple policy dimensions. Chicago: Midwest Political Science Association.

Brady, Henry E., Sidney Verba, und Kay L. Schlozman. 1995. Beyond SES: a resource model of political participation. The American Political Science Review 89(2):271-294.

Breunig, Christian, und Benjamin Guinaudeau. 2019. German Policy Agenda. Deutsch-Englische Version des internationalen CAP Codebook.

Canes-Wrone, Brandice, und Kenneth W. Shotts. 2004. The conditional nature of presidential responsiveness to public opinion. American Journal of Political Science 48(4):690-706.

Carmines, Edward G., und James A. Stimson. 1986. On the structure and sequence of issue evolution. American Political Science Review 80(3):901-920.

Citrin, Jack, und Laura Stoker. 2018. Political trust in a cynical age. Annual Review of Political Science 21:49-70.

Claassen, Ryan L., und Stephen P. Nicholson. 2013. Extreme voices. Interest groups and the misrepresentation of issue publics. Public Opinion Quarterly 77(4):861-887.

Dahl, Robert A. 1971. Polyarchy: participation and opposition. New Haven: Yale University Press.

Dahl, Robert A. 1989. Democracy and its critics. New Haven: Yale University Press.

De Lange, Sarah L. 2007. A new winning formula? The programmatic appeal of the radical right. Party Politics 13(4):411-435.

Drope, Jeffrey M., und Wendy L. Hansen. 2006. Does firm size matter? Analyzing business lobbying in the United States. Business and Politics 10(2):1-17.

Dür, Andreas, Patrick Bernhagen, und David Marshall. 2015. Interest Group Success in the European Union: When (and Why) does Business Lose? Comparative Political Studies 48(8):951-983.

Easton, David. 1971. Political system. An inquiry into the state of political science. New York: Alfred A. Knopf.

Eising, Rainer, und Florian Spohr. 2016. The more, the merrier? Interest groups and legislative change in the public hearings of the German parliamentary committees. German Politics 26(2):1-20.

Elsässer, Lea, Svenja Hense, und Armin Schäfer. 2017. „Dem Deutschen Volke“? Die ungleiche Responsivität des Bundestags. Zeitschrift für Politikwissenschaft 27(2):161-180. 
Erikson, Robert S., Gerald C. Wright, und John P. McIver. 1993. Statehouse democracy: public opinion and policy in the American states. Cambridge: Cambridge University Press.

Flöthe, Linda, und Anne Rasmussen. 2019. Public voices in the heavenly chorus? Group type bias and opinion representation. Journal of European Public Policy 26(6):824-842.

Franke, James L., und Douglas Dobson. 1985. Interest groups. The problem of representation. The Western Political Quarterly 38(2):224-237.

Giger, Nathalie, Jan Rosset, und Julian Bernauer. 2012. The poor political representation of the poor in a comparative perspective. Representation 48(1):47-61.

Gilens, Martin. 2012. Affluence and influence. Economic inequality and political power in America. Princeton: Princeton University Press.

Gilens, Martin, und Benjamin I. Page. 2014. Testing theories of American politics: elites, interest groups, and average citizens. Perspectives on Politics 12(3):564-581.

Golder, Matt, und Benjamin Ferland. 2018. Electoral systems and citizen-elite ideological congruence. In The oxford handbook of electoral systems, Hrsg. Erik S. Herron, Robert J. Pekkanen, und Matthew S. Shugart, 213-246. Oxford: Oxford University Press.

Gray, Virginia, und David Lowery. 1996. A niche theory of interest representation. The Journal of Politics 58(1):91-111.

Grier, Kevin B., Michael C. Munger, und Brian E. Roberts. 1994. The determinants of industry political activity, 1978-1986. The American Political Science Review 88(4):911-926.

Halpin, Darren R., Bert Fraussen, und Anthony J. Nownes. 2018. The balancing act of establishing a policy agenda: conceptualizing and measuring drivers of issue prioritization within interest groups. Governance 31(2):215-237.

Jones, Bryan D., und Frank R. Baumgartner. 2004. Representation and Agenda-Setting. The Policy Studies Journal 32(1):1-24.

Jungar, Ann-Cathrine, und Anders R. Jupskas. 2014. Populist radical right parties in the nordic region: a new and distinct party family? Scandinavian Political Studies 37(3):215-238.

Key, Valdimer O. 1962. Politics, parties, \& pressure groups. New York: Crowell.

Kimball, David C., Frank R. Baumgartner, Jeffrey M. Berry, Marie Hojnacki, Beth L. Leech, und Bryce Summary. 2012. Who cares about the lobbying agenda? Interest Groups \& Advocacy 1(1):5-25.

Klüver, Heike. 2015. Interest groups in the German Bundestag: exploring the issue linkage between citizens and interest groups. German Politics 24(2):137-153.

Klüver, Heike. 2018. Setting the party agenda: interest groups, voters and issue attention. British Journal of Political Science. https://doi.org/10.1017/S0007123418000078.

Kollman, Ken. 1998. Outside lobbying. Public opinion and interest group strategies. Princeton: Princeton University Press.

Kuklinski, James H. 1978. Representativeness and elections: a policy analysis. American Political Science Review 72(1):165-177.

Lax, Jeffrey R., und Justin H. Phillips. 2012. The democratic deficit in the states. American Journal of Political Science 56(1):148-166.

Lefkofridi, Zoe, Markus Wagner, und Johanna E. Willmann. 2014. Left-authoritarians and policy representation. Western europe: electoral choice across ideological dimensions. West European Politics 37(1):65-90.

Lijphart, Arend. 2012. Patterns of democracy. Government forms and performance in thirty-six countries. New Haven: Yale University Press.

Lowery, David. 2007. Why do organized interests lobby? A multi-goal, multi-context theory of lobbying. Polity 39(1):29-54.

McCarthy, John D., und Mayer N. Zald. 1977. Resource mobilization and social movements: a partial theory. American Journal of Sociology 82(6):1212-1241.

Monroe, Alan D. 1979. Consistency between public preferences and national policy decisions. American Politics Quarterly 7(1):3-19.

Monroe, Alan D. 1998. Public opinion and public policy, 1980-1993. The Public Opinion Quarterly 62(1):6-28.

Mudde, Cas. 1996. The paradox of the anti-party party: insights from the extreme right. Party Politics 2(2):265-276.

Newton, Kenneth. 2012. Curing the democratic malaise with democratic innovations. In Evaluating democratic innovations. Curing the democratic malaise?, Hrsg. Kenneth Newton, Brigitte Geissel, 3-20. New York: Routledge.

Norris, Pippa. 2011. Democratic deficit. Critical citizens revisited. Cambridge: Cambridge University Press. 
Olson, Mancur. 1965. The Logic of Collective Action: Public Goods and the Theory of Groups. Cambridge: Harvard University Press.

Peters, Yvette, und Sander J. Ensink. 2015. Differential responsiveness in Europe: the effects of preference difference and electoral participation. West European Politics 38(3):577-600.

Rasmussen, Anne, Brendan J. Carroll, und David Lowery. 2014. Representatives of the public? Public opinion and interest group activity. European Journal of Political Research 53(2):250-268.

Rasmussen, Anne, Lars Kai Mäder, und Stefanie Reher. 2017. With a little help from the people? The role of public opinion in advocacy success. Comparative Political Studies 51(2):139-164.

Rauh, Christian. 2016. A responsive technocracy. EU Politicisation and the consumer policies of the European commission. Colchester: ECPR Press.

Richardson, Jeremy S. 1995. The market for political activism: interest groups as a challenge to political parties. West European Politics 18(1):116-139.

Romeijn, Jeroen. 2018. Do political parties listen to the(ir) public? Public opinion-party linkage on specific policy issues. Party Politics. https://doi.org/10.1177/1354068818787346.

Sabatier, Paul A., und Susan M. McLaughlin. 1990. Belief congruence between interest-group leaders and members: an empirical analysis of three theories and a suggested synthesis. Journal of Politics 52(3):914-935.

Schattschneider, Elmer E. 1960. The Semisovereign people. A realist's view of democracy in America. New York: Wadsworth Publishing.

Schlozman, Kay L., und John T. Tierney. 1986. Organized Interests and American Democracy. New York: Harper \& Row.

Spoon, Jae-Jae, und Heike Klüver. 2014. Do parties respond? How electoral context influences party responsiveness. Electoral Studies 35:48-60.

Stecker, Christian, und Markus Tausendpfund. 2016. Multidimensional government-citizen congruence and satisfaction with democracy. European Journal of Political Research 55(3):492-511.

Stimson, James A., Michael B. Mackuen, und Robert S. Erikson. 1995. Dynamic representation. American Political Science Review 89(3):543-565.

Thaidigsmann, Isabell S. 2000. Parteien und Verbände als Vertreter von Bürgerinteressen. In Wirklich ein Volk? Die politischen Orientierungen von Ost- und Westdeutschen im Vergleich, Hrsg. Jürgen Falter, Oscar W. Gabriel, und Hans Rattinger, 9-38. Opladen: Leske + Budrich.

Truman, David B. 1951. The governmental process: political interests and public opinion. New York: Alfred A. Knopf.

Van der Brug, Wouter, und Joost van Spanje. 2009. Immigration, europe and the 'new' cultural dimension. European Journal of Political Research 48(3):309-334.

Verba, Sidney, und Norman H. Nie. 1972. Participation in America. Political democracy and social equality. Chicago London: University of Chicago Press.

Verba, Sidney, Kay L. Schlozman, und Henry E. Brady. 1995. Voice and equality. Civic voluntarism in American politics. Cambridge: Harvard University Press.

Von Beyme, Klaus. 2017. Das politische System der Bundesrepublik Deutschland: Eine Einführung. Wiesbaden: Springer VS.

Williams, Melissa S. 2000. The uneasy alliance of group representation and deliberative democracy. In Citizenship in diverse societies, Hrsg. Will Kymlicka, Wayne Norman, 124-154. Oxford: Oxford University Press.

Wilson, James Q. 1995. Political organizations. New York: Basic Books.

Wlezien, Christopher. 1995. The responsive public. Issue salience, policy change, and preferences for European unification. American Journal of Political Science 39(4):347-363.

Wlezien, Christopher. 2004. Patterns of representation: dynamics of public preferences and policy. Journal of Politics 66(1):1-24.

Wlezien, Christopher. 2005. On the salience of political issues: the problem with 'most important problem. Electoral Studies 24(4):555-579.

Wlezien, Christopher. 2017. Public opinion and policy representation: on conceptualization, measurement, and interpretation. Policy Studies Journal 45(4):561-582.

Wlezien, Christopher, und Stuart N. Soroka. 2012. Political institutions and the opinion-policy link. West European Politics 35(6):1407-1432. 